\title{
Solving the Faddeev-Merkuriev Equations in Total Orbital Momentum Representation via Spline Collocation and Tensor Product Preconditioning
}

\author{
Vitaly A. Gradusov ${ }^{1, *}$, Vladimir A. Roudnev ${ }^{1}$, Evgeny A. Yarevsky ${ }^{1}$ and \\ Sergey L. Yakovlev ${ }^{1}$ \\ ${ }^{1}$ Department of Computational Physics, St Petersburg State University, \\ St Petersburg 199034, Russia.
}

Received 25 May 2020; Accepted (in revised version) 18 November 2020

\begin{abstract}
The computational approach for solving the Faddeev-Merkuriev equations in total orbital momentum representation is presented. These equations describe a system of three quantum charged particles and are widely used in bound state and scattering calculations. The approach is based on the spline collocation method and exploits intensively the tensor product form of discretized operators and preconditioner, which leads to a drastic economy in both computer resources and time.
\end{abstract}

AMS subject classifications: 65F08, 65N35, 81Q05, 81U35

Key words: Faddeev-Merkuriev equations, total orbital momentum representation, spline collocation, tensor product preconditioner.

\section{Introduction}

Since the pioneering work of Hylleraas [1], quantum three-body systems remain the source of challenges and inspirations for theoretical and experimental physicists. New effects specific to three-body systems have been predicted, such as Thomas effect [2], Efimov effect [3], the Phillips line [4,5]. Direct modeling of nuclear and molecular threebody systems paved a way to develop and to fine-tune realistic models of inter-atomic and inter-nucleon interactions [6-9]. Ab-initio calculations of some specific three-atomic systems may give essential contributions to metrology [10]. The Coulomb quantum three-body systems are also of great importance. For instance, delicate calculations of asymmetric heavy-hydrogen molecular ions gave an insight on $m u$-catalysis [11], studies of positron-atom interactions are valuable for positron-emission tomography.

*Corresponding author. Email addresses: v.gradusov@spbu.ru (V. A. Gradusov), v.roudnev@spbu.ru (V. A. Roudnev), e.yarevsky@spbu.ru (E. A. Yarevsky), s.yakovlev@spbu.ru (S. L. Yakovlev) 
Even though the basic mathematical model for such a broad spectrum of physical systems is the Schrödinger equation, the diversity of model interactions and particular physical states leads to a variety of employed computational methods [12-25]. Thus, our ability to perform direct model-free calculations for such wide range of systems is of utmost importance for many branches of physics.

Our goal is to present a universal and efficient computational framework applicable to this broad variety of physical systems and states. In order to achieve this goal we start from the following presuppositions. The approach should be based on a physically correct and mathematically sound representation of the problem. The Faddeev equations formalism [26-28] fulfills all of these requirements. Clear separation of asymptotic channels corresponding to different clusterisations of the system is one of the main advantageous features of the formalism from the point of view of practical applications. Coulomb systems are incorporated into the original formalism by the Merkuriev's version of the Faddeev equations in $[29,30]$, where the splitting of the Coulomb potentials into long-rage and short-range parts was introduced. Being mathematically equivalent to the Schrödinger equation [29,30], the Faddeev-Merkuriev (FM) equations have advantages of much simpler boundary conditions and much simpler behavior of their solutions. This leads to much weaker requirements for the basis employed in the calculations.

Direct solution of the FM equations is not, however, a simple task. In order to reduce the dimensionality of the configuration space the symmetries of the solutions must be taken into account. We base our computational approach on total orbital momentum representation which leads to systems of partial differential equations in three-dimensional space. Solving such systems numerically is still a challenging task which calls for developing an effective and robust preconditioning technique. Here we propose a preconditioning scheme based on the tensor-trick algorithm and compare our numerical scheme with solving the corresponding sparse linear system using PARDISO direct solver. Our approach clearly outperforms the direct method both in time and memory requirements, which paves a way to accurate calculations of rather challenging systems, including highly rotationally excited three-body states.

In the following sections we give a description of the FM equations formalism, describe our numerical scheme, and give a few computational examples for some wellstudied systems of diverse physical nature.

Throughout the paper we assume $\hbar=1$ and we use bold font for vectors as, for instance, $x$ and normal font for their magnitude $x=|x|$.

\section{The Faddeev-Merkuriev equations}

\subsection{Notation and basic equations}

The FM equations for three quantum particles are of the form 


$$
\begin{aligned}
& \left\{T_{\alpha}+V_{\alpha}\left(x_{\alpha}\right)+\sum_{\beta \neq \alpha} V_{\beta}^{(1)}\left(x_{\beta}, y_{\beta}\right)-E\right\} \psi_{\alpha}\left(x_{\alpha}, y_{\alpha}\right) \\
= & -V_{\alpha}^{(\mathrm{s})}\left(x_{\alpha}, y_{\alpha}\right) \sum_{\beta \neq \alpha} \psi_{\beta}\left(x_{\beta}, y_{\beta}\right), \quad \alpha=1,2,3 .
\end{aligned}
$$

These equations describe bound and scattering states with the energy $E$ of a system of three spinless non-relativistic charged particles of masses $m_{\alpha}$ and charges $Z_{\alpha}$ in their center of mass system of coordinates [30]. In what follows the indices $\alpha, \beta, \gamma$ run over the set $\{1,2,3\}$ enumerating particles and an index, say $\alpha$, is also used for identifying the complementary pair of particles, since in the partition $\{\alpha(\beta \gamma)\}$ the pair of particles $\beta \gamma$ is uniquely determined by the particle with the number $\alpha$. The particle positions are described by pairs of Jacobi vectors (see Fig. 1). The standard Jacobi coordinates are defined for a partition $\alpha(\beta \gamma)$ as the relative position vector $x_{\alpha}$ between the particles of the pair $\beta \gamma$ and the relative vector $y_{\alpha}$ between their center of mass and the particle $\alpha$. In applications it is convenient to use reduced Jacobi coordinates $x_{\alpha}, \boldsymbol{y}_{\alpha}$ which are Jacobi vectors scaled by factors $\sqrt{2 \mu_{\alpha}}$ and $\sqrt{\left.2 \mu_{\alpha(\beta \gamma)}\right)}$, respectively, where the reduced masses are given by

$$
\mu_{\alpha}=\frac{m_{\beta} m_{\gamma}}{m_{\beta}+m_{\gamma}}, \quad \mu_{\alpha(\beta \gamma)}=\frac{m_{\alpha}\left(m_{\beta}+m_{\gamma}\right)}{m_{\alpha}+m_{\beta}+m_{\gamma}} .
$$

For different $\alpha^{\prime} s$ the reduced Jacobi vectors are related by an orthogonal transform

$$
\boldsymbol{x}_{\beta}=c_{\beta \alpha} \boldsymbol{x}_{\alpha}+s_{\beta \alpha} \boldsymbol{y}_{\alpha}, \quad \boldsymbol{y}_{\beta}=-s_{\beta \alpha} \boldsymbol{x}_{\alpha}+c_{\beta \alpha} \boldsymbol{y}_{\alpha} \text {, }
$$

where

$$
c_{\beta \alpha}=-\left[\frac{m_{\beta} m_{\alpha}}{\left(M-m_{\beta}\right)\left(M-m_{\alpha}\right)}\right]^{1 / 2}, \quad s_{\beta \alpha}=(-1)^{\beta-\alpha} \operatorname{sgn}(\beta-\alpha)\left(1-c_{\beta \alpha}^{2}\right)^{1 / 2}
$$

and $M=\sum_{\alpha} m_{\alpha}$. In what follows, where it is due, it is assumed that $\beta$ Jacobi vectors are represented through $\alpha$ vectors via (2.3). The orientation of Jacobi vectors is important, and it is chosen as it is shown in Fig. 1. The kinetic energy operators are given by $T_{\alpha} \equiv-\Delta_{x_{\alpha}}-\Delta_{y_{\alpha}}$. The potentials $V_{\alpha}$ represent a sum of the pairwise Coulomb interaction $V_{\alpha}^{\mathrm{C}}\left(x_{\alpha}\right)=\sqrt{2 \mu_{\alpha}} Z_{\beta} Z_{\gamma} / x_{\alpha}(\beta, \gamma \neq \alpha)$ and a short-range $V_{\alpha}^{\text {sh }}\left(x_{\alpha}\right)$ (decreasing faster than $1 / x_{\alpha}^{2}$ as $\left.x_{\alpha} \rightarrow \infty\right)$ potential. The potentials $V_{\alpha}$ are split into the interior (short-range) $V_{\alpha}^{(\mathrm{s})}$ and the tail (long-range) parts $V_{\alpha}^{(1)}$

$$
V_{\alpha}\left(x_{\alpha}\right)=V_{\alpha}^{(\mathrm{s})}\left(x_{\alpha}, y_{\alpha}\right)+V_{\alpha}^{(1)}\left(x_{\alpha}, y_{\alpha}\right) .
$$

Eqs. (2.1) can be summed up leading to the Schrödinger equation for the wave function $\Psi=\sum_{\alpha} \psi_{\alpha}$, where $\psi_{\alpha}$ are the wave function components given by the solution of Eqs. (2.1). 


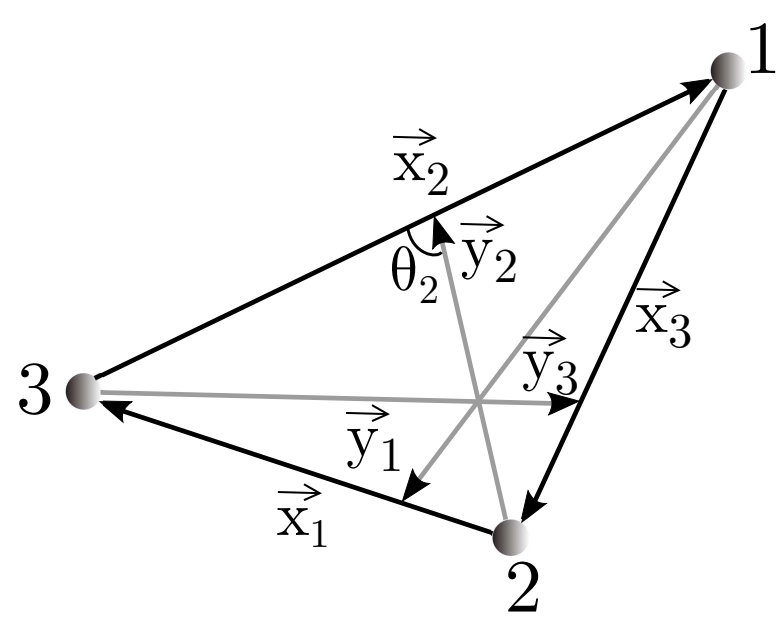

Figure 1: Jacobi coordinates for three particles.

The splitting procedure (2.4) is especially important for scattering calculations. It makes the properties of the FM equations for the Coulomb potentials as appropriate for scattering problems as the standard Faddeev equations in the case of short-range potentials $[19,30]$. The key property of the FM Eqs. (2.1) is that the right-hand side of each equation is a square-integrable function (i.e. confined to the vicinity of the triple collision point) [30,31]. Splitting (2.4) of the potentials in general case is done in the three-body configuration space by the Merkuriev cut-off function $\chi_{\alpha}$ [30]

$$
V_{\alpha}^{(\mathrm{s})}\left(x_{\alpha}, y_{\alpha}\right)=\chi_{\alpha}\left(x_{\alpha}, y_{\alpha}\right) V_{\alpha}^{\mathrm{C}}\left(x_{\alpha}\right)+V_{\alpha}^{\mathrm{sh}}\left(x_{\alpha}\right), \quad V_{\alpha}^{(1)}\left(x_{\alpha}, y_{\alpha}\right)=\left(1-\chi_{\alpha}\left(x_{\alpha}, y_{\alpha}\right)\right) V_{\alpha}^{\mathrm{C}}\left(x_{\alpha}\right) .
$$

This splitting confines the short-range part of the potential to the regions in the threebody configuration space corresponding to the three-body collision point (particles are close to each other) and the binary configuration $\left(x_{\alpha} \ll y_{\alpha}\right.$, as $\left.y_{\alpha} \rightarrow \infty\right)$. The form of the cut-off function can be rather arbitrary within some general requirements [29]. One of the most often used variant [32] of this function reads

$$
\chi_{\alpha}\left(x_{\alpha}, y_{\alpha}\right)=2 /\left\{1+\exp \left[\left(x_{\alpha} / x_{0 \alpha}\right)^{v_{\alpha}} /\left(1+y_{\alpha} / y_{0 \alpha}\right)\right]\right\} .
$$

The parameters $x_{0 \alpha}, y_{0 \alpha}$ and $v_{\alpha}>2$ can in principle be chosen arbitrarily, but their choice changes the properties of components $\psi_{\alpha}$ that are important from both theoretical and computational points of view [31]. In the papers [33,34] we have discussed the choice of the cut-off function and have presented some practical algorithm of how to choose its parameters efficiently. In the bound state calculations the splitting can be omitted, that is one can take $V_{\alpha}^{(\mathrm{s})}=V_{\alpha}$ and $V_{\alpha}^{(1)}=0$. Then Eqs. (2.1) turn into standard Faddeev equations. On the other hand, in many calculations the choice $V_{\beta}^{(\mathrm{s})}=0, V_{\beta}^{(1)}=V_{\beta}$ with $\beta=3$ or $\beta=2,3$ is possible [33]. Then the set of Eqs. (2.1) decouples leading to two equations in the first case, and to the Schrödinger equation in the second case. Therefore in principle, the methods of this paper are applicable to solving the Schrödinger equation as well. 
Asymptotic boundary conditions are to be added to Eqs. (2.1). The bound state calculations imply zero Dirichlet-type boundary conditions $\psi_{\alpha}\left(x_{\alpha}, y_{\alpha}\right) \rightarrow 0$ as $x_{\alpha}$ or $y_{\alpha} \rightarrow \infty$. The resulting eigenvalue problem allows one to determine the discrete energy spectrum. The scattering problem gives rise to much more complicated radiation boundary conditions and a subsequent boundary value problem. The exact asymptotic form of each component depends on the total energy $E$. Comprehensive descriptions of scattering boundary conditions can be found in [30] (for the total energy $E$ of the system below the three-body breakup threshold see also formula (8) of [33] and discussion therein). Summarizing, for Eqs. (2.1) two problems are possible:

- determination of discrete energy levels of bound states: eigenvalue problem with Eqs. (2.1) and asymptotic zero Dirichlet-type boundary conditions;

- scattering problem at a given total energy $E$ : boundary value problem with Eqs. (2.1) and asymptotic boundary conditions for scattering states.

The methods that we describe in this paper are equally applicable for solving both problems, but for definiteness in what follows we refer to the bound state problem.

\subsection{Total orbital momentum representation. Kinematics}

Eqs. (2.1) are six dimensional PDE and therefore their direct solving on the modern computers is quite a challenging problem. One of the ways to decrease the dimension of the problem is to use some basis expansion for the solution $\psi_{\alpha}$. Among the approaches used for calculations, an expansion in bipolar harmonics is often used [18,35,36], which reduces (2.1) to an infinite set of coupled two dimensional integro-differential equations. This set is then truncated to the finite set of equations to make their numerical solution possible. In [37] another approach was proposed. It is based on the expansion of components $\psi_{\alpha}$ in terms of eigenfunctions of the total orbital momentum squared operator. The total orbital momentum is an integral of motion for the three-particle system. This makes it possible to reduce the FM equations to a finite set of three dimensional (3D) PDE by projecting onto a subspace of a given total orbital momentum. These equations are the 3D FM equations in total orbital momentum representation and are the equations that we solve in this work. In the subsequent part of this section we briefly outline the derivation of the 3D FM equations by using a notation more appropriate for our purposes than in [37].

We start by introducing new kinematic coordinates $\left(X_{\alpha}, \Omega_{\alpha}\right)$ in the six dimensional configuration space of the problem. The coordinates $X_{\alpha}=\left\{x_{\alpha}, y_{\alpha}, z_{\alpha}\right\}$ determine particle positions in the plane which contains all three particles, $z_{\alpha} \equiv\left(\boldsymbol{x}_{\alpha}, \boldsymbol{y}_{\alpha}\right) /\left(x_{\alpha} y_{\alpha}\right)$ is cosine of an angle $\theta_{\alpha}$ between the vectors $x_{\alpha}$ and $y_{\alpha}$. They are depicted in Fig. 1 . The coordinates $\Omega_{\alpha}=\left\{\phi_{\alpha}, \vartheta_{\alpha}, \varphi_{\alpha}\right\}$ determine the orientation of the plain containing the particles. They are defined as follows: let $x y z$ be some laboratory system of coordinates and $x^{\prime \prime \prime} y^{\prime \prime \prime} z^{\prime \prime \prime}$ the body-fixed system of coordinates in which $\boldsymbol{y}_{\alpha}$ is along the $z^{\prime \prime \prime}$-axis and $\boldsymbol{x}_{\alpha}$ lies in the 


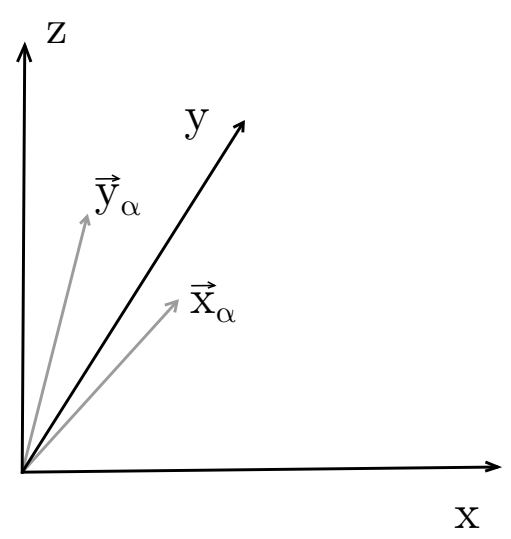

(a)

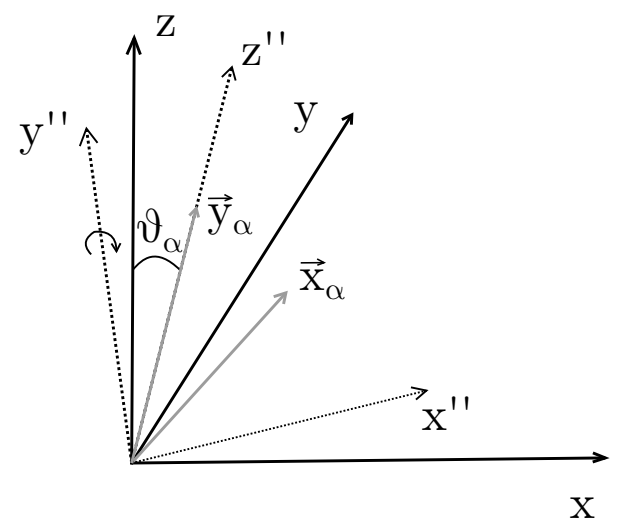

(c)

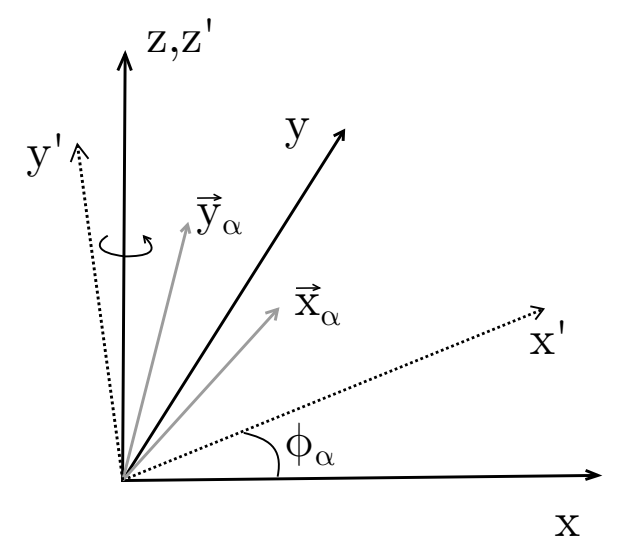

(b)

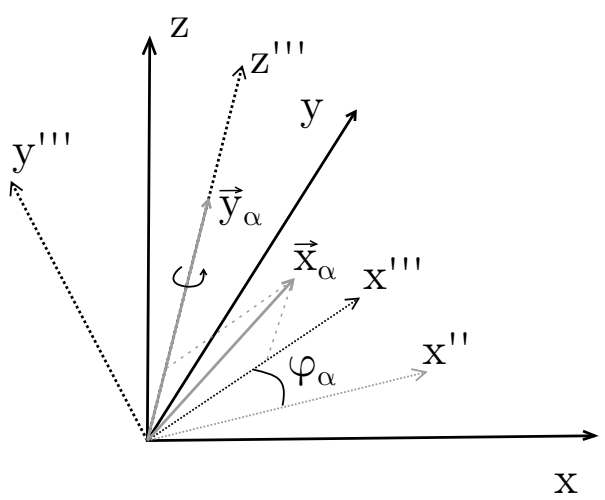

(d)

Figure 2: Definition of Euler angles $\phi_{\alpha}, \vartheta_{\alpha}, \varphi_{\alpha}$.

$x^{\prime \prime \prime} z^{\prime \prime \prime}$-plane. They are depicted in Fig. 2(d). Then $\phi_{\alpha}, \vartheta_{\alpha}, \varphi_{\alpha}$ are the Euler angles of rotation of the laboratory to the body-fixed system of coordinates. Three rotations are being done: the rotation in counter-clockwise direction around the $z$-axis by an angle $\phi_{\alpha} \in[0,2 \pi)$ is followed by the rotation in counter-clockwise direction around the new $y^{\prime}$-axis by an angle $\vartheta_{\alpha} \in[0, \pi)$, and the last rotation in counter-clockwise direction is around the new $z^{\prime \prime}$-axis by an angle $\varphi_{\alpha} \in[0,2 \pi$ ) (Fig. 2). These rotations are described by the standard rotation matrix [38]

$$
\begin{aligned}
& \mathfrak{R}\left(\phi_{\alpha}, \vartheta_{\alpha}, \varphi_{\alpha}\right) \\
= & \left(\begin{array}{ccc}
\cos \phi_{\alpha} \cos \vartheta_{\alpha} \cos \varphi_{\alpha}-\sin \phi_{\alpha} \sin \varphi_{\alpha} & -\cos \phi_{\alpha} \cos \vartheta_{\alpha} \sin \varphi_{\alpha}-\sin \phi_{\alpha} \cos \varphi_{\alpha} & \cos \phi_{\alpha} \sin \vartheta_{\alpha} \\
\sin \phi_{\alpha} \cos \vartheta_{\alpha} \cos \varphi_{\alpha}+\cos \phi_{\alpha} \sin \varphi_{\alpha} & -\sin \phi_{\alpha} \cos \vartheta_{\alpha} \sin \varphi_{\alpha}+\cos \phi_{\alpha} \cos \varphi_{\alpha} & \sin \phi_{\alpha} \sin \vartheta_{\alpha} \\
-\sin \vartheta_{\alpha} \cos \varphi_{\alpha} & \sin \vartheta_{\alpha} \sin \varphi_{\alpha} & \cos \vartheta_{\alpha}
\end{array}\right) .
\end{aligned}
$$


Now the connection between sets of coordinates $\left(\boldsymbol{x}_{\alpha}, \boldsymbol{y}_{\alpha}\right)$ and $\left(X_{\alpha}, \Omega_{\alpha}\right)$ is easily expressed by

$$
\begin{aligned}
& \boldsymbol{x}_{\alpha}=x_{\alpha} \mathfrak{R}\left(\phi_{\alpha}, \vartheta_{\alpha}, \varphi_{\alpha}\right)\left(\begin{array}{c}
\sin \theta_{\alpha} \\
0 \\
\cos \theta_{\alpha}
\end{array}\right), \quad \boldsymbol{y}_{\alpha}=y_{\alpha} \mathfrak{R}\left(\phi_{\alpha}, \vartheta_{\alpha}, \varphi_{\alpha}\right)\left(\begin{array}{l}
0 \\
0 \\
1
\end{array}\right), \\
& \tan \phi_{\alpha}=\frac{\left(\boldsymbol{y}_{\alpha}\right)_{2}}{\left(\boldsymbol{y}_{\alpha}\right)_{1}}, \quad \cos \vartheta_{\alpha}=\frac{\left(\boldsymbol{y}_{\alpha}\right)_{3}}{y_{\alpha}}, \quad \cos \varphi_{\alpha}=\frac{-\left(\boldsymbol{x}_{\alpha}\right)_{3} / x_{\alpha}+\cos \vartheta_{\alpha} \cos \theta_{\alpha}}{\sin \vartheta_{\alpha} \sin \theta_{\alpha}} .
\end{aligned}
$$

In the case of formulae (2.9) the ambiguity of angle values is resolved by the direction of the rotations described by $\mathfrak{R}\left(\phi_{\alpha}, \vartheta_{\alpha}, \varphi_{\alpha}\right)$. The connection between different sets of coordinates $X_{\alpha}$ and $X_{\beta}$ easily follows from (2.3):

$$
\begin{aligned}
& x_{\beta}=\sqrt{c_{\beta \alpha}^{2} x_{\alpha}^{2}+2 s_{\beta \alpha} c_{\beta \alpha} x_{\alpha} y_{\alpha} z_{\alpha}+s_{\beta \alpha}^{2} y_{\alpha}^{2}}, \\
& y_{\beta}=\sqrt{s_{\beta \alpha}^{2} x_{\alpha}^{2}-2 s_{\beta \alpha} c_{\beta \alpha} x_{\alpha} y_{\alpha} z_{\alpha}+c_{\beta \alpha}^{2} y_{\alpha}^{2}}, \\
& z_{\beta}=\frac{s_{\beta \alpha} c_{\beta \alpha}\left(y_{\alpha}^{2}-x_{\alpha}^{2}\right)+\left(c_{\beta \alpha}^{2}-s_{\beta \alpha}^{2}\right) x_{\alpha} y_{\alpha} z_{\alpha}}{x_{\beta} y_{\beta}} .
\end{aligned}
$$

The connection between sets of Euler angles can be written in the form

$$
\mathfrak{R}\left(\phi_{\beta}, \vartheta_{\beta}, \varphi_{\beta}\right)=\mathfrak{R}\left(\phi_{\alpha}, \vartheta_{\alpha}, \varphi_{\alpha}\right) \mathfrak{R}\left(0, w_{\beta \alpha}, 0\right),
$$

where $w_{\beta \alpha}$ is the angle such that the rotation by this angle in the plain containing particles places vector $y_{\beta}$ in the position of vector $y_{\alpha}$. Eq. (2.11) follows immediately from the identity $\mathfrak{R}^{-1}\left(0, w_{\beta \alpha}, 0\right) \mathfrak{R}^{-1}\left(\phi_{\alpha}, \vartheta_{\alpha}, \varphi_{\alpha}\right)=\mathfrak{R}^{-1}\left(\phi_{\beta}, \vartheta_{\beta}, \varphi_{\beta}\right)$ which simply expresses the fact that coordinates of a vector in the body-fixed frames defined with respect to different Jacobi coordinate sets $\alpha$ and $\beta$ are connected by the rotation matrix $\mathfrak{R}\left(0, w_{\beta \alpha}, 0\right)$. With the orientation of Jacobi vectors chosen in this article (see Fig. 1) the angle $w_{\beta \alpha}$ is given by

$$
w_{\beta \alpha}= \begin{cases}\arccos \frac{-s_{\beta \alpha} x_{\alpha} z_{\alpha}+c_{\beta \alpha} y_{\alpha}}{y_{\beta}}, & \text { if }(\beta, \alpha)=(2,1),(3,2),(1,3), \\ 2 \pi-\arccos \frac{-s_{\beta \alpha} x_{\alpha} z_{\alpha}+c_{\beta \alpha} y_{\alpha}}{y_{\beta}}, & \text { otherwise, }\end{cases}
$$

where the range of arccos is $[0, \pi]$.

\subsection{Total orbital momentum representation. Equations}

The change of variables in Eqs. (2.1) can be derived quite easily with any symbolic computing system like Mathematica [39]. The equations now read

$$
\begin{aligned}
& \left\{T_{\alpha}+V_{\alpha}\left(x_{\alpha}\right)+\sum_{\beta \neq \alpha} V_{\beta}^{(1)}\left(x_{\beta}, y_{\beta}\right)-E\right\} \psi_{\alpha}\left(X_{\alpha}, \Omega_{\alpha}\right) \\
= & -V_{\alpha}^{(\mathrm{s})}\left(x_{\alpha}, y_{\alpha}\right) \sum_{\beta \neq \alpha} \psi_{\beta}\left(X_{\beta}, \Omega_{\beta}\right), \quad \alpha=1,2,3,
\end{aligned}
$$


where $\psi_{\alpha}\left(X_{\alpha}, \Omega_{\alpha}\right)$ denote the components expressed in new variables. The kinetic energy operator in new variables takes the form

$$
\begin{aligned}
T_{\alpha}= & -\frac{1}{y_{\alpha}^{2}} \frac{\partial}{\partial y_{\alpha}} y_{\alpha}^{2} \frac{\partial}{\partial y_{\alpha}}-\frac{1}{x_{\alpha}^{2}} \frac{\partial}{\partial x_{\alpha}} x_{\alpha}^{2} \frac{\partial}{\partial x_{\alpha}} \\
& -\left(\frac{1}{y_{\alpha}^{2}}+\frac{1}{x_{\alpha}^{2}}\right)\left(\frac{1}{\sin \theta_{\alpha}} \frac{\partial}{\partial \theta_{\alpha}} \sin \theta_{\alpha} \frac{\partial}{\partial \theta_{\alpha}}+\frac{1}{\sin ^{2} \theta_{\alpha}} \frac{\partial^{2}}{\partial \varphi_{\alpha}^{2}}\right)+\frac{J^{2}-K_{\alpha}}{y_{\alpha}^{2}},
\end{aligned}
$$

where $J^{2}$ is the total orbital momentum squared operator

$$
J^{2}=-\left[\frac{1}{\sin \vartheta_{\alpha}} \frac{\partial}{\partial \vartheta_{\alpha}} \sin \vartheta_{\alpha} \frac{\partial}{\partial \vartheta_{\alpha}}+\frac{1}{\sin ^{2} \vartheta_{\alpha}}\left(\frac{\partial^{2}}{\partial \phi_{\alpha}^{2}}-2 \cos \vartheta_{\alpha} \frac{\partial^{2}}{\partial \phi_{\alpha} \partial \varphi_{\alpha}}+\frac{\partial^{2}}{\partial \varphi_{\alpha}^{2}}\right)\right]
$$

and $\boldsymbol{K}_{\alpha}$ is given by

$$
\boldsymbol{K}_{\alpha}=\frac{\partial}{\partial \theta_{\alpha}}\left(\boldsymbol{J}_{\alpha}^{(+)}+\boldsymbol{J}_{\alpha}^{(-)}\right)+\cot \theta_{\alpha}\left(\boldsymbol{J}_{\alpha}^{(+)}-\boldsymbol{J}_{\alpha}^{(-)}\right) \boldsymbol{J}_{z^{\prime}}+2 \boldsymbol{J}_{z^{\prime}}^{2}
$$

with

$$
J_{\alpha}^{( \pm)}=\mp \mathrm{e}^{\mp \mathrm{i} \varphi_{\alpha}}\left[ \pm \frac{\partial}{\partial \vartheta_{\alpha}}+\frac{\mathrm{i}}{\sin \vartheta_{\alpha}} \frac{\partial}{\partial \phi_{\alpha}}-\mathrm{i} \cot \vartheta_{\alpha} \frac{\partial}{\partial \varphi_{\alpha}}\right], \quad J_{z^{\prime}}=-\mathrm{i} \partial / \partial \varphi_{\alpha} .
$$

Now we introduce Wigner $D$-functions $D_{M, M^{\prime}}^{J}$

$$
D_{M M^{\prime}}^{J}\left(\phi_{\alpha}, \vartheta_{\alpha}, \varphi_{\alpha}\right)=\mathrm{e}^{-\mathrm{i} M \phi_{\alpha}} d_{M M^{\prime}}^{J}\left(\vartheta_{\alpha}\right) \mathrm{e}^{-\mathrm{i} M^{\prime} \varphi_{\alpha}},
$$

where

$$
d_{M M^{\prime}}^{J}\left(\vartheta_{\alpha}\right)=\sqrt{\frac{\left(J+M^{\prime}\right) !(J-M) !}{(J+M) !(J-M) !}}\left(\sin \frac{\vartheta_{\alpha}}{2}\right)^{M^{\prime}-M}\left(\cos \frac{\vartheta_{\alpha}}{2}\right)^{M^{\prime}+M} P_{J-M^{\prime}}^{\left(M^{\prime}-M, M^{\prime}+M\right)}\left(\cos \vartheta_{\alpha}\right) .
$$

Here $P_{n}^{(\alpha, \beta)}$ are the Jacobi polynomials [40]. The definition (2.18) coincides with those of $[41,42]$, but differs from that of [37]. Wigner $D$-functions are the common eigenfunctions of total orbital momentum squared $J^{2}$ and its projection $J_{z}=-\mathrm{i} \partial / \partial \phi_{\alpha}$ operators

$$
J^{2} D_{M M^{\prime}}^{J}=J(J+1) D_{M M^{\prime \prime}}^{J} \quad J_{z} D_{M M^{\prime}}^{J}=-M D_{M M^{\prime}}^{J}
$$

They also obey

$$
J_{\alpha}^{( \pm)} D_{M M^{\prime}}^{J}= \pm \lambda^{J, \pm M^{\prime}} D_{M M^{\prime} \pm 1^{\prime}}^{J} \quad J_{z^{\prime}} D_{M M^{\prime}}^{J}=-M^{\prime} D_{M M^{\prime \prime}}^{J}
$$

where

$$
\lambda^{J M^{\prime}}=\sqrt{J(J+1)-M^{\prime}\left(M^{\prime}+1\right)}
$$


The orthogonality conditions are

$$
\begin{aligned}
& \int_{0}^{2 \pi} \mathrm{d} \phi_{\alpha} \int_{0}^{2 \pi} \mathrm{d} \varphi_{\alpha} \int_{0}^{\pi} \mathrm{d} \vartheta_{\alpha} \sin \vartheta_{\alpha}\left(D_{M_{1} M_{1}^{\prime}}^{J_{1}}\left(\phi_{\alpha}, \vartheta_{\alpha}, \varphi_{\alpha}\right)\right)^{*} D_{M_{2} M_{2}^{\prime}}^{J_{2}}\left(\phi_{\alpha}, \vartheta_{\alpha}, \varphi_{\alpha}\right) \\
= & \frac{8 \pi^{2}}{2 J_{1}+1} \delta_{J_{1} J_{2}} \delta_{M_{1} M_{2}} \delta_{M_{1}^{\prime} M_{2}^{\prime}} .
\end{aligned}
$$

Due to the rotation transformation (2.11) the Wigner D-functions of arguments with different index $\alpha$ are bound by the following relationship

$$
D_{M M^{\prime}}^{J}\left(\phi_{\beta}, \vartheta_{\beta}, \varphi_{\beta}\right)=\sum_{M^{\prime \prime}=-J}^{J} D_{M M^{\prime \prime}}^{J}\left(\phi_{\alpha}, \vartheta_{\alpha}, \varphi_{\alpha}\right) D_{M^{\prime \prime} M^{\prime}}^{J}\left(0, w_{\beta \alpha}, 0\right) .
$$

Wigner $D$-functions with integer $J \geq 0$ and $|M|,\left|M^{\prime}\right| \leq J$ form a basis in the space of square integrable functions on the domain $[0,2 \pi] \times[0, \pi] \times[0,2 \pi]$. However, as a basis for the expansion of FM components we use linear combinations of Wigner $D$-functions defined as

$$
F_{M M^{\prime}}^{J \tau}\left(\Omega_{\alpha}\right)=\frac{1}{\sqrt{2+2 \delta_{M^{\prime} 0}}}\left(D_{M M^{\prime}}^{J}\left(\Omega_{\alpha}\right)+\tau(-1)^{M^{\prime}} D_{M,-M^{\prime}}^{J}\left(\Omega_{\alpha}\right)\right),
$$

where $\tau= \pm 1$ is the parity. They are constructed to be the eigenfunctions not only of $J^{2}$ and $J_{z}$ but also of the inversion operator

$$
\boldsymbol{P} F_{M M^{\prime}}^{J \tau}\left(\phi_{\alpha}, \vartheta_{\alpha}, \varphi_{\alpha}\right)=F_{M M^{\prime}}^{J \tau}\left(\phi_{\alpha}+\pi, \pi-\vartheta_{\alpha}, \pi-\varphi_{\alpha}\right)=\tau(-1)^{J} F_{M M^{\prime}}^{J \tau}\left(\phi_{\alpha}, \vartheta_{\alpha}, \varphi_{\alpha}\right) .
$$

This equation follows easily from the identity $D_{M M^{\prime}}^{J}\left(\phi_{\alpha}+\pi, \pi-\vartheta_{\alpha}, \pi-\varphi_{\alpha}\right)=$ $(-1)^{J+M^{\prime}} D_{M,-M^{\prime}}^{J}\left(\phi_{\alpha}, \vartheta_{\alpha}, \varphi_{\alpha}\right)$. Eqs. (2.20), (2.21) for $F_{M M^{\prime}}^{J \tau}$ become

$$
\begin{gathered}
J^{2} F_{M M^{\prime}}^{J \tau}=J(J+1) F_{M M^{\prime}}^{J \tau}, \quad J_{z} F_{M M^{\prime}}^{J \tau}=-M F_{M M^{\prime \prime}}^{J \tau}, \quad J_{z^{\prime}} F_{M M^{\prime}}^{J \tau}=-M^{\prime} F_{M M^{\prime \prime}}^{J,-\tau}, \\
\left(J_{\alpha}^{(+)}+J_{\alpha}^{(-)}\right) F_{M M^{\prime}}^{J \tau}=\lambda^{J, M^{\prime}} F_{M, M^{\prime}+1}^{J \tau} \sqrt{1+\delta_{M^{\prime} 0}}\left(1-\delta_{M^{\prime} 0} \delta_{\tau,-1}\right) \\
-\lambda^{J,-M^{\prime}} F_{M, M^{\prime}-1}^{J \tau} \sqrt{1+\delta_{M^{\prime} 1}}\left(1-\delta_{M^{\prime} 0}\right)\left(1-\delta_{M^{\prime} 1} \delta_{\tau,-1}\right), \\
\left(J_{\alpha}^{(+)}-J_{\alpha}^{(-)}\right) J_{z^{\prime}} F_{M M^{\prime}}^{J \tau}=-M^{\prime} \lambda^{J, M^{\prime}} F_{M, M^{\prime}+1}^{J \tau} \sqrt{1+\delta_{M^{\prime} 0}}\left(1-\delta_{M^{\prime} 0} \delta_{\tau,-1}\right) \\
-M^{\prime} \lambda^{J,-M^{\prime}} F_{M, M^{\prime}-1}^{J \tau} \sqrt{1+\delta_{M^{\prime} 1}}\left(1-\delta_{M^{\prime} 0}\right)\left(1-\delta_{M^{\prime} 1} \delta_{\tau,-1}\right) .
\end{gathered}
$$

The orthogonality conditions are easily deduced from (2.23)

$$
\begin{aligned}
& \int_{0}^{2 \pi} \mathrm{d} \phi_{\alpha} \int_{0}^{2 \pi} \mathrm{d} \varphi_{\alpha} \int_{0}^{\pi} \mathrm{d} \vartheta_{\alpha} \sin \vartheta_{\alpha}\left(F_{M_{1} M_{1}^{\prime}}^{J_{1} \tau_{1}}\left(\phi_{\alpha}, \vartheta_{\alpha}, \varphi_{\alpha}\right)\right)^{*} F_{M_{2} M_{2}^{\prime}}^{J_{2} \tau_{2}}\left(\phi_{\alpha}, \vartheta_{\alpha}, \varphi_{\alpha}\right) \\
= & \frac{8 \pi^{2}}{2 J_{1}+1} \delta_{J_{1} J_{2}} \delta_{\tau_{1} \tau_{2}} \delta_{M_{1} M_{2}} \delta_{M_{1}^{\prime} M_{2}^{\prime}}
\end{aligned}
$$


and the relation between the functions of different arguments follows from (2.24)

$$
F_{M M^{\prime}}^{J \tau}\left(\phi_{\beta}, \vartheta_{\beta}, \varphi_{\beta}\right)=\sum_{M^{\prime \prime}=M_{0}}^{J}(-1)^{M^{\prime}-M^{\prime \prime}} \frac{2}{\sqrt{2+2 \delta_{M^{\prime} 0}}} F_{M^{\prime} M^{\prime \prime}}^{J \tau}\left(0, w_{\beta \alpha}, 0\right) F_{M M^{\prime \prime}}^{J \tau}\left(\phi_{\alpha}, \vartheta_{\alpha}, \varphi_{\alpha}\right),
$$

where we have defined

$$
M_{0}=(1-\tau) / 2
$$

Finally, from the definition (2.25) and the identity $F_{M,-M^{\prime}}^{J \tau}=\tau(-1)^{M^{\prime}} F_{M, M^{\prime}}^{J \tau}$ it follows that the functions $F_{M M^{\prime}}^{J \tau}$ with integer $J \geq 0, \tau= \pm 1,|M| \leq J$ and $(1-\tau) / 2 \leq M^{\prime} \leq J$ also form a basis in the space of square integrable functions on the domain $[0,2 \pi] \times[0, \pi] \times[0,2 \pi]$.

We are now ready to expand the FM components in terms of the functions $F_{M M^{\prime}}^{J \tau}$

$$
\psi_{\alpha}\left(X_{\alpha}, \Omega_{\alpha}\right)=\sum_{J=0}^{+\infty} \sum_{\tau= \pm 1} \sum_{M=-J M^{\prime}=M_{0}}^{J} \frac{\psi_{\alpha M M^{\prime}}^{J \tau}\left(X_{\alpha}\right)}{x_{\alpha} y_{\alpha}} F_{M M^{\prime}}^{J \tau}\left(\phi_{\alpha}, \vartheta_{\alpha}, \varphi_{\alpha}\right) .
$$

The factor $1 /\left(x_{\alpha} y_{\alpha}\right)$ is introduced here to get rid of the first derivatives in $x_{\alpha}$ and $y_{\alpha}$ in the resulting equations. Substituting (2.31) into the FM Eqs. (2.1), projecting them onto the basis functions $F_{M M^{\prime}}^{J \tau}$ and using Eqs. (2.27)-(2.29) we obtain the finite set of 3D equations for partial components $\psi_{\alpha M M^{\prime}}^{J \tau}\left(X_{\alpha}\right)$

$$
\begin{aligned}
& {\left[T_{\alpha M M^{\prime}}^{J \tau}+V_{\alpha}\left(x_{\alpha}\right)+\sum_{\beta \neq \alpha} V_{\beta}^{(1)}\left(x_{\beta}, y_{\beta}\right)-E\right] \psi_{\alpha M M^{\prime}}^{J \tau}\left(X_{\alpha}\right)} \\
& \quad+T_{\alpha M, M^{\prime}-1}^{J \tau-} \psi_{\alpha M, M^{\prime}-1}^{J \tau}\left(X_{\alpha}\right)+T_{\alpha M, M^{\prime}+1}^{J \tau+} \psi_{\alpha M, M^{\prime}+1}^{J \tau}\left(X_{\alpha}\right) \\
& =-V_{\alpha}^{(\mathrm{s})}\left(x_{\alpha}, y_{\alpha}\right) \sum_{\beta \neq \alpha} \frac{x_{\alpha} y_{\alpha}}{x_{\beta} y_{\beta}} \sum_{M^{\prime \prime}=M_{0}}^{J}(-1)^{M^{\prime \prime}-M^{\prime}} \frac{2}{\sqrt{2+2 \delta_{M^{\prime \prime} 0}}} F_{M^{\prime \prime} M^{\prime}}^{J \tau}\left(0, w_{\beta \alpha}, 0\right) \psi_{\beta M M^{\prime \prime}}^{J \tau}\left(X_{\beta}\right) .
\end{aligned}
$$

Here the kinetic part is of the form

$$
\begin{aligned}
T_{\alpha M M^{\prime}}^{J \tau}= & -\frac{\partial^{2}}{\partial y_{\alpha}^{2}}+\frac{1}{y_{\alpha}^{2}}\left(J(J+1)-2 M^{\prime 2}\right) \\
& -\frac{\partial^{2}}{\partial x_{\alpha}^{2}}-\left(\frac{1}{y_{\alpha}^{2}}+\frac{1}{x_{\alpha}^{2}}\right)\left(\frac{\partial}{\partial z_{\alpha}}\left(1-z_{\alpha}^{2}\right) \frac{\partial}{\partial z_{\alpha}}-\frac{M^{\prime 2}}{1-z_{\alpha}^{2}}\right), \\
T_{\alpha M, M^{\prime} \pm 1}^{J \tau \pm}= & \pm \frac{1}{y_{\alpha}^{2}} \lambda^{J, \pm M^{\prime}} \sqrt{1+\delta_{M^{\prime} 0(1)}}\left[-\sqrt{1-z_{\alpha}^{2}} \frac{\partial}{\partial z_{\alpha}} \pm\left(M^{\prime} \pm 1\right) \frac{z_{\alpha}}{\sqrt{1-z_{\alpha}^{2}}}\right] .
\end{aligned}
$$

The key property of the obtained equations is that equations with different $J, \tau$ and $M$ form independent sets of equations. This is the direct consequence of the fact that the total orbital momentum, its projection and the parity are the integrals of motion for the threeparticle system. For given $J, \tau$ and $M$ Eqs. (2.32) are enumerated by indices $M^{\prime}=M_{0}, \cdots, J$ and $\alpha=1,2,3$ thus forming a finite set of $3 n_{M}$ three dimensional PDEs. Here

$$
n_{M}=J-M_{0}+1
$$


is the number of possible values of $M^{\prime}$ for given $J$ and $\tau$. In addition to zero Dirichlet asymptotic boundary conditions the partial components $\psi_{\alpha M M^{\prime}}^{J \tau}$ must also satisfy zero boundary conditions on the lines $x_{\alpha}=0, y_{\alpha}=0$ to be continuous.

Eqs. (2.32) are called the (3D) FM equations in total orbital momentum representation. For the first time they were obtained in [37] in a slightly different form. They are the main result of this section.

\subsection{Permutational symmetry}

As is well known from the basics of quantum mechanics, presence of identical particles in the system leads to a symmetry (antisymmetry) of the wave function. Here we show how it can be used to reduce the number of 3D FM equations. In the three-particle system two cases of two or three identical particles are possible. Let the particles $\alpha$ and $\beta$ be identical. Then the operator $P_{\alpha \beta}$ which permutes the coordinates of particles $\alpha$ and $\beta$ in a wave function is an additional integral of motion for a three-particle system and commutes with the full Hamiltonian of a system. As a result, equations for symmetric and antisymmetric parts of the wave function can be solved independently.

Let $\psi_{\alpha}$ be the FM components of a symmetric or antisymmetric part of the wave function $\Psi=\psi_{1}+\psi_{2}+\psi_{3}$ satisfying $P_{\alpha \beta} \Psi=p \Psi$ with $p=1$ or $p=-1$ respectively. We now find the constraints on the components in these cases. Consider for definiteness the symmetry (antisymmetry) with respect to operator $P_{12}$. Note that permutation of particles 1 and 2 leads to the following change in pairs of Jacobi vectors:

$$
\left(x_{1}, y_{1}\right) \rightarrow\left(-x_{2}, y_{2}\right), \quad\left(x_{2}, y_{2}\right) \rightarrow\left(-x_{1}, y_{1}\right), \quad\left(x_{3}, y_{3}\right) \rightarrow\left(-x_{3}, y_{3}\right) .
$$

Then from the relation

$$
\begin{aligned}
P_{12}\left(\psi_{1}\left(\boldsymbol{x}_{1}, \boldsymbol{y}_{1}\right)+\psi_{2}\left(\boldsymbol{x}_{2}, \boldsymbol{y}_{2}\right)+\psi_{3}\left(\boldsymbol{x}_{3}, \boldsymbol{y}_{3}\right)\right) & =\psi_{1}\left(-\boldsymbol{x}_{2}, \boldsymbol{y}_{2}\right)+\psi_{2}\left(-\boldsymbol{x}_{1}, \boldsymbol{y}_{1}\right)+\psi_{3}\left(-\boldsymbol{x}_{3}, \boldsymbol{y}_{3}\right) \\
& =p \psi_{1}\left(\boldsymbol{x}_{1}, \boldsymbol{y}_{1}\right)+p \psi_{2}\left(\boldsymbol{x}_{2}, \boldsymbol{y}_{2}\right)+p \psi_{3}\left(\boldsymbol{x}_{3}, \boldsymbol{y}_{3}\right)
\end{aligned}
$$

we obtain the constraints

$$
\psi_{2}\left(x_{2}, \boldsymbol{y}_{2}\right)=p \psi_{1}\left(-\boldsymbol{x}_{2}, \boldsymbol{y}_{2}\right), \quad \psi_{3}\left(\boldsymbol{x}_{3}, \boldsymbol{y}_{3}\right)=p \psi_{3}\left(-\boldsymbol{x}_{3}, \boldsymbol{y}_{3}\right) .
$$

Thus in the case of two identical particles 1 and 2 the component $\psi_{3}$ is symmetric (antisymmetric) with respect to its argument $x_{3}$ and components $\psi_{1}$ and $\psi_{2}$ are simply related by the first identity of (2.38). In the case of three identical particles and fully (anti)symmetric wave function it obeys $P_{12} \Psi=P_{23} \Psi=P_{13} \Psi=p \Psi$, then additionally we have

$$
\psi_{1}\left(\boldsymbol{x}_{1}, \boldsymbol{y}_{1}\right)=\psi_{2}\left(\boldsymbol{x}_{1}, \boldsymbol{y}_{1}\right)=\psi_{3}\left(\boldsymbol{x}_{1}, \boldsymbol{y}_{1}\right)=p \psi_{1}\left(-\boldsymbol{x}_{1}, \boldsymbol{y}_{1}\right) .
$$

In coordinates $\left(X_{\alpha}, \Omega_{\alpha}\right)$ the substitution $\left(\boldsymbol{x}_{\alpha}, \boldsymbol{y}_{\alpha}\right) \rightarrow\left(-\boldsymbol{x}_{\alpha}, \boldsymbol{y}_{\alpha}\right)$ reads

$$
\left(x_{\alpha}, y_{\alpha}, z_{\alpha}, \phi_{\alpha}, \vartheta_{\alpha}, \varphi_{\alpha}\right) \rightarrow\left(x_{\alpha}, y_{\alpha},-z_{\alpha}, \phi_{\alpha}, \vartheta_{\alpha},\left\{\begin{array}{l}
\varphi_{\alpha}+\pi, \varphi_{\alpha} \in[0, \pi), \\
\varphi_{\alpha}-\pi, \varphi_{\alpha} \in[\pi, 2 \pi)
\end{array}\right) .\right.
$$


Then from the identity $F_{M M^{\prime}}^{J \tau}\left(\phi_{\alpha}, \vartheta_{\alpha}, \varphi_{\alpha} \pm \pi\right)=(-1)^{M^{\prime}} F_{M M^{\prime}}^{J \tau}\left(\phi_{\alpha}, \vartheta_{\alpha}, \varphi_{\alpha}\right)$ and the definition of partial components (2.31) we get

$$
\psi_{\alpha}\left(-\boldsymbol{x}_{\alpha}, \boldsymbol{y}_{\alpha}\right)=\sum_{J \tau M M^{\prime}}(-1)^{M^{\prime}} \frac{\psi_{\alpha M M^{\prime}}^{J \tau}\left(x_{\alpha}, y_{\alpha},-z_{\alpha}\right)}{x_{\alpha} y_{\alpha}} F_{M M^{\prime}}^{J \tau}\left(\phi_{\alpha}, \vartheta_{\alpha}, \varphi_{\alpha}\right) .
$$

Thus, finally, the constraints (2.38) and (2.39) for partial components take the form

$$
\begin{aligned}
& \psi_{2 M M^{\prime}}^{J \tau}\left(x_{2}, y_{2}, z_{2}\right)=p(-1)^{M^{\prime}} \psi_{1 M M^{\prime}}^{J \tau}\left(x_{2}, y_{2},-z_{2}\right), \\
& \psi_{3 M M^{\prime}}^{J \tau}\left(x_{3}, y_{3}, z_{3}\right)=p(-1)^{M^{\prime}} \psi_{3 M M^{\prime}}^{J \tau}\left(x_{3}, y_{3},-z_{3}\right)
\end{aligned}
$$

and

$$
\begin{aligned}
\psi_{1 M M^{\prime}}^{J \tau}\left(x_{1}, y_{1}, z_{1}\right) & =\psi_{2 M M^{\prime}}^{J \tau}\left(x_{1}, y_{1}, z_{1}\right)=\psi_{3 M M^{\prime}}^{J \tau}\left(x_{1}, y_{1}, z_{1}\right) \\
& =p(-1)^{M^{\prime}} \psi_{1 M M^{\prime}}^{J \tau}\left(x_{1}, y_{1},-z_{1}\right) .
\end{aligned}
$$

The relations (2.42) and (2.43) allow one to solve Eqs. (2.32) only for components $\psi_{\alpha M M^{\prime}}^{J \tau}$ with $\alpha=1,3$ in the case of two identical particles and with $\alpha=1$ in the case of three identical particles. The number of coupled three-dimensional equations is then reduced from $3 n_{M}$ to $2 n_{M}$ and $n_{M}$, respectively. Moreover, the (anti)symmetry of functions $\psi_{3 M M^{\prime}}^{J \tau}$ and $\psi_{1 M M^{\prime}}^{J \tau}$ in (2.42) and (2.43) reduces the $z_{\alpha}$-domain in corresponding equations to $z_{\alpha} \in[0,1]$.

\section{The computational scheme}

\subsection{Basic scheme}

We start this section by transforming Eqs. (2.32) by making a substitution

$$
\psi_{\alpha M M^{\prime}}^{J \tau}\left(x_{\alpha}, y_{\alpha}, z_{\alpha}\right)=\left(1-z_{\alpha}^{2}\right)^{\frac{M^{\prime}}{2}} \widehat{\psi}_{\alpha M M^{\prime}}^{J \tau}\left(x_{\alpha}, y_{\alpha}, z_{\alpha}\right) .
$$

The reason for that is as follows. It can be shown [43] that the singular term $M^{\prime 2} /\left(1-z_{\alpha}^{2}\right)$ in operator $T_{\alpha M M^{\prime}}^{J \tau}$ enforces the partial components to have the following behaviour at $z_{\alpha}= \pm 1$

$$
\left.\psi_{\alpha M M^{\prime}}^{J \tau}\left(x_{\alpha}, y_{\alpha}, z_{\alpha}\right)\right|_{z_{\alpha} \rightarrow \pm 1} \sim\left(1-z_{\alpha}^{2}\right)^{\frac{M^{\prime}}{2}}
$$

It makes problematic the use of smooth bases for the expansion of partial components with odd $M^{\prime}$. Substitution (3.1) corrects it. The new partial components $\widehat{\psi}_{\alpha M M^{\prime}}^{J \tau}$ obey the 
equations

$$
\begin{aligned}
& {\left[\widehat{T}_{\alpha M M^{\prime}}^{J \tau}+V_{\alpha}\left(x_{\alpha}\right)+\sum_{\beta \neq \alpha} V_{\beta}^{(1)}\left(x_{\beta}, y_{\beta}\right)-E\right] \widehat{\psi}_{\alpha M M^{\prime}}^{J \tau}\left(X_{\alpha}\right) } \\
& +\widehat{T}_{\alpha M, M^{\prime}-1}^{J \tau-} \widehat{\psi}_{\alpha M, M^{\prime}-1}^{J \tau}\left(X_{\alpha}\right)+\widehat{T}_{\alpha M, M^{\prime}+1}^{J \tau+} \widehat{\psi}_{\alpha M, M^{\prime}+1}^{J \tau}\left(X_{\alpha}\right) \\
=- & \frac{V_{\alpha}^{(s)}\left(x_{\alpha}, y_{\alpha}\right)}{\left(1-z_{\alpha}^{2}\right)^{\frac{M^{\prime}}{2}}} \sum_{\beta \neq \alpha} \frac{x_{\alpha} y_{\alpha}}{x_{\beta} y_{\beta}} \sum_{M^{\prime \prime}=M_{0}}^{J}(-1)^{M^{\prime \prime}-M^{\prime}} \frac{2}{\sqrt{2+2 \delta_{M^{\prime \prime} 0}}} \\
& \times F_{M^{\prime \prime} M^{\prime}}^{J \tau}\left(0, w_{\beta \alpha}, 0\right)\left(1-z_{\beta}^{2}\right)^{\frac{M^{\prime \prime}}{2}} \widehat{\psi}_{\beta M M^{\prime \prime}}^{J \tau}\left(X_{\beta}\right),
\end{aligned}
$$

where the new kinetic part operators are

$$
\begin{aligned}
\widehat{T}_{\alpha M M^{\prime}}^{J \tau}= & -\frac{\partial^{2}}{\partial y_{\alpha}^{2}}+\frac{1}{y_{\alpha}^{2}}\left(J(J+1)-2 M^{\prime 2}\right)-\frac{\partial^{2}}{\partial x_{\alpha}^{2}} \\
& -\left(\frac{1}{y_{\alpha}^{2}}+\frac{1}{x_{\alpha}^{2}}\right)\left(\left(1-z_{\alpha}^{2}\right) \frac{\partial^{2}}{\partial z_{\alpha}^{2}}-2\left(M^{\prime}+1\right) z_{\alpha} \frac{\partial}{\partial z_{\alpha}}-M^{\prime}\left(M^{\prime}+1\right)\right), \\
\widehat{T}_{\alpha M, M^{\prime}+1}^{J \tau+}= & \frac{1}{y_{\alpha}^{2}} \widehat{\lambda}^{J M^{\prime}}\left[-\left(1-z_{\alpha}^{2}\right) \frac{\partial}{\partial z_{\alpha}}+2\left(M^{\prime}+1\right) z_{\alpha}\right], \\
\widehat{T}_{\alpha M, M^{\prime}-1}^{J \tau-}= & \frac{1}{y_{\alpha}^{2}} \widehat{\lambda}^{J,-M^{\prime}} \frac{\partial}{\partial z_{\alpha}},
\end{aligned}
$$

where we have introduced

$$
\widehat{\lambda}^{J, \pm M^{\prime}}=\lambda^{J, \pm M^{\prime}} \sqrt{1+\delta_{M^{\prime} 0(1)}}
$$

Now we make the last but not least step of preparing the 3D FM equations for discretization. For that we represent the sum of the tail parts of potentials at the left hand side of equations in the form

$$
\sum_{\beta \neq \alpha} V_{\beta}^{(1)}\left(x_{\beta}, y_{\beta}\right)=\widehat{V}_{\alpha}\left(y_{\alpha}\right)+\left(\sum_{\beta \neq \alpha} V_{\beta}^{(1)}\left(x_{\beta}, y_{\beta}\right)-\widehat{V}_{\alpha}\left(y_{\alpha}\right)\right)
$$

and rearrange terms in equations so that they become

$$
\begin{aligned}
& {\left[\widehat{T}_{\alpha M M^{\prime}}^{J \tau}+V_{\alpha}\left(x_{\alpha}\right)+\widehat{V}_{\alpha}\left(y_{\alpha}\right)-E\right] \widehat{\psi}_{\alpha M M^{\prime}}^{J \tau}\left(X_{\alpha}\right) } \\
& +\widehat{T}_{\alpha M, M^{\prime}-1}^{\tau-} \widehat{\psi}_{\alpha M, M^{\prime}-1}^{J \tau}\left(X_{\alpha}\right)+\widehat{T}_{\alpha M, M^{\prime}+1}^{\tau \tau+} \widehat{\psi}_{\alpha M, M^{\prime}+1}^{J \tau}\left(X_{\alpha}\right) \\
= & -\frac{V_{\alpha}^{\mathrm{s}}\left(x_{\alpha}, y_{\alpha}\right)}{\left(1-z_{\alpha}^{2}\right)^{\frac{M^{\prime}}{2}}} \sum_{\beta \neq \alpha} \frac{x_{\alpha} y_{\alpha}}{x_{\beta} y_{\beta}} \sum_{M^{\prime \prime}=M_{0}}^{J}(-1)^{M^{\prime \prime}-M^{\prime}} \frac{2}{\sqrt{2+2 \delta_{M^{\prime \prime} 0}}} \\
& \times F_{M^{\prime \prime} M^{\prime}}^{J \tau}\left(0, w_{\beta \alpha}, 0\right)\left(1-z_{\beta}^{2}\right)^{\frac{M^{\prime}}{2}} \widehat{\psi}_{\beta M M^{\prime \prime}}^{J \tau}\left(X_{\beta}\right) \\
& -\left(\sum_{\beta \neq \alpha} V_{\beta}^{(1)}\left(x_{\beta}, y_{\beta}\right)-\widehat{V}_{\alpha}\left(y_{\alpha}\right)\right) \widehat{\psi}_{\alpha M, M^{\prime}}^{J \tau}\left(X_{\alpha}\right) .
\end{aligned}
$$


The potential $\widehat{V}_{\alpha}$ is an approximation of $\sum_{\beta \neq \alpha} V_{\beta}^{(1)}$ in the sense that the new right hand side term $\left(\sum_{\beta \neq \alpha} V_{\beta}^{(1)}-\widehat{V}_{\alpha}\right) \widehat{\psi}_{\alpha M, M^{\prime}}^{I \tau}$ is small. For better theoretical and computational properties of the resulting equations the potential $\widehat{V}_{\alpha}$ 's choice must secure the square integrability of this term. The actual form depends on the problem that is solved. For bound states and below the breakup threshold scattering calculations the possible choice is $\widehat{V}_{\alpha}\left(y_{\alpha}\right)=$ $Z_{\alpha}\left(Z_{\beta}+Z_{\gamma}\right) \sqrt{2 \mu_{\alpha(\beta \gamma)}} /\left(y_{\alpha}+1\right)$ (this is the leading term of $\sum_{\beta \neq \alpha} V_{\beta}^{(1)}$ when $y_{\alpha} \rightarrow+\infty$ with $x_{\alpha}$ fixed). Eqs. (3.8) are the final form of 3D FM equations in total orbital momentum representation that are discretized. The reason for the rearrangement done is that now variables "almost separate" in the operator at the left hand side and it can be used to construct an effective preconditioner in the computational scheme.

In a nutshell, our scheme is based on the collocation method with local basis. It results in the generalized eigenvalue problem of the form

$$
H c=E S c .
$$

To find eigenvalues in the vicinity of a given $E=E^{*}$ it is rewritten in the form

$$
S\left(H-E^{*} S\right)^{-1} \widetilde{\boldsymbol{c}}=\frac{1}{E-E^{*}} \widetilde{\boldsymbol{c}}
$$

with $\widetilde{\boldsymbol{c}}=\left(H-E^{*} S\right) \boldsymbol{c}$. Then an iterative method is applied to find the eigenvalues of the matrix $S\left(H-E^{*} S\right)^{-1}$. For solving systems of linear equations with the matrix $\left(H-E^{*} S\right)$ we use a preconditioning matrix which is an approximation of the inverse matrix of the operator at the left-hand side of Eqs. (3.8) with $E=E^{*}$. This choice of a preconditioner is exploited in $[35,36,44]$. In the calculations presented in Section 4 we use a local basis of splines. However, the results presented in Subsections 3.2 and 3.3 remain true for any basis within some, presented below, requirements on it. We note that there we don't deal with the convergence properties of the collocation method applied to 3D FM equations. Naturally, the specific choice of a basis affects the convergence. See the discussion in Subsection 3.4 below.

Let the functions $S_{\alpha}^{i}(x), S_{\alpha}^{j}(y)$ and $S_{\alpha}^{k}(z)$ form bases of local functions defined on given intervals $\left[0, R_{x_{\alpha}}\right],\left[0, R_{y_{\alpha}}\right]$ and $[-1,1]$ and $S_{\alpha}^{i}(x), S_{\alpha}^{j}(y)$ satisfy zero Dirichlet-type boundary conditions at the end points. Denote by $r$ the overlap rate, i.e. the highest possible number of basis functions that are nonzero at any point of interval of definition for all sets $S_{\alpha}^{i}$, $S_{\alpha}^{j}$ and $S_{\alpha}^{k}$. We seek the solutions $\widehat{\psi}_{\alpha M M^{\prime}}^{j \tau}$ of (3.8) in the cubes $\left[0, R_{x_{\alpha}}\right] \times\left[0, R_{y_{\alpha}}\right] \times[-1,1]$ expanding them in terms of products of those basis functions

$$
\widehat{\psi}_{\alpha M M^{\prime}}^{J \tau}\left(x_{\alpha}, y_{\alpha}, z_{\alpha}\right)=\sum_{i, j, k=1}^{n_{x_{\alpha}}, n_{y_{\alpha}}, n_{z_{\alpha}}} c_{i j k}^{M^{\prime}} S_{\alpha}^{i}\left(x_{\alpha}\right) S_{\alpha}^{j}\left(y_{\alpha}\right) S_{\alpha}^{k}\left(z_{\alpha}\right) .
$$

We use the same basis set for partial components $\widehat{\psi}_{\alpha M M^{\prime}}^{\tau}$ with different $M^{\prime}$. This fact is not used explicitly in constructing the scheme which can be easily generalized on the case of $M^{\prime}$ dependent basis sets. 
Let us introduce regular grids of collocation points $\left(x_{\alpha}^{\tau}, y_{\alpha}^{\eta}, z_{\alpha}^{\zeta}\right)$ with $\xi=1, \cdots, n_{x_{\alpha}}$, $\eta=1, \cdots, n_{y_{\alpha}}, \zeta=1, \cdots, n_{z_{\alpha}}$. Again for notation simplicity we use identical sets of collocation points for Eqs. (3.8) with different $M^{\prime}$. Requiring the Eqs. (3.8) to be satisfied at collocation points, we obtain the generalized eigenvalue problem (3.10). The collocation matrix $H$ is the discretized version of 3D FM Eqs. (3.8) operator with $E=0$ and $S$ is the matrix with elements $S_{\alpha}^{i}\left(x_{\alpha}^{\tau}\right) S_{\alpha}^{j}\left(y_{\alpha}^{\eta}\right) S_{\alpha}^{k}\left(z_{\alpha}^{\zeta}\right)$. The obtained matrix $H$ has linear dimension $n_{M} \sum_{\alpha} n_{x_{\alpha}} n_{y_{\alpha}} n_{z_{\alpha}}$. However, due to the use of local bases the number of nonzero elements of $H$ is $\mathcal{O}\left(n_{M}^{2} \sum_{\alpha} n_{x_{\alpha}} n_{y_{\alpha}} n_{z_{\alpha}}\right)$ only.

\subsection{Preconditioner}

We now turn to construction of the preconditioner that allows us to efficiently invert the operator at the left-hand side of Eqs. (3.8). For that we use tensor product methods based on the technique that is known as Matrix Decomposition Algorithm (MDA) [45] or Tensor Trick (TT) [35]. Let us first outline some preliminary facts. The Kronecker product of $n \times n$ matrix $A$ and $m \times m$ matrix $B$ is the $(n m) \times(n m)$ matrix $A \otimes B$ with elements $(A \otimes$ $B)_{(i-1) m+k,(j-1) m+l}=a_{i j} b_{k l}$. The following properties are satisfied:

1.

$$
\left(A_{1} \otimes B_{1}\right)\left(A_{2} \otimes B_{2}\right)=\left(A_{1} A_{2}\right) \otimes\left(B_{1} B_{2}\right) ;
$$

2.

$$
(A \otimes B)^{-1}=A^{-1} \otimes B^{-1} .
$$

MDA (TT) is based on the following trick. Suppose we need to invert the $(\mathrm{nm}) \times(\mathrm{nm})$ matrix of the form

$$
A_{1} \otimes B_{1}+A_{2} \otimes B_{2}
$$

with $n \times n$ matrices $A_{1,2}$ and $m \times m$ matrices $B_{1,2}$. Let now $\bar{W}_{A}, W_{A}$ and $\Lambda_{A}$ be (if exist) nonsingular complex matrices which are the solutions of left and right generalized eigenvalue problems

$$
\bar{W}_{A} A_{1}=\Lambda_{A} \bar{W}_{A} A_{2}, \quad A_{1} W_{A}=A_{2} W_{A} \Lambda_{A} .
$$

Then the matrices $\bar{W}_{A}$ and $W_{A}$ simultaneously diagonalize matrices $A_{1}$ and $A_{2}$. They can be scaled to satisfy

$$
\bar{W}_{A} A_{1} W_{A}=\Lambda_{A}, \quad \bar{W}_{A} A_{2} W_{A}=I,
$$

where $I$ is the identity matrix of the proper size. In the following we denote the operation of finding the solutions of generalized eigenvalue problems that satisfy (3.16) by $\mathfrak{D}$

$$
\left(\bar{W}_{A}, W_{A}, \Lambda_{A}\right)=\mathfrak{D}\left(A_{1}, A_{2}\right) .
$$

We note that the computational cost of $\mathfrak{D}$ is $\mathcal{O}\left(n^{3}\right)$. Now let also

$$
\left(\bar{W}_{B}, W_{B}, \Lambda_{B}\right)=\mathfrak{D}\left(B_{1}, B_{2}\right) .
$$


Then one obtains

$$
A_{1} \otimes B_{1}+A_{2} \otimes B_{2}=\left(\bar{W}_{A}^{-1} \otimes \bar{W}_{B}^{-1}\right)\left(\Lambda_{A} \otimes \Lambda_{B}+I \otimes I\right)\left(W_{A}^{-1} \otimes W_{B}^{-1}\right)
$$

and consequently

$$
\left(A_{1} \otimes B_{1}+A_{2} \otimes B_{2}\right)^{-1}=\left(W_{A} \otimes W_{B}\right)\left(\Lambda_{A} \otimes \Lambda_{B}+I \otimes I\right)^{-1}\left(\bar{W}_{A} \otimes \bar{W}_{B}\right) .
$$

The matrix $\Lambda_{A} \otimes \Lambda_{B}+I \otimes I$ is diagonal and is inverted fast. With the use of (3.20) the inversion of the matrix requires $\mathcal{O}\left(n^{3}+m^{3}\right)$ instead of $\mathcal{O}\left(n^{3} m^{3}\right)$ operations if treating it as a matrix of general form. The matrix-vector product with the matrix of the form (3.20) requires $\mathcal{O}(n m(n+m))$ multiplications instead of the ordinary $\mathcal{O}\left(n^{2} m^{2}\right)$. The review of theory and applications of MDAs can be found in [45].

We denote the discretized version of the operator at the left-hand side of Eqs. (3.8) with $E=E^{*}$ by $L$. This matrix is block diagonal with blocks $L_{\alpha}$ that can be inverted independently. Consider the $L_{\alpha}$ block. It is block tridiagonal with blocks $L_{\alpha M^{\prime}, M^{\prime}-1}$, $L_{\alpha M^{\prime} M^{\prime}}$ and $L_{\alpha M^{\prime}, M^{\prime}+1}$ in row $M^{\prime}=M_{0}, \cdots, J$. Let the collocation points $\left(x_{\alpha}^{\tau}, y_{\alpha}^{\eta}, z_{\alpha}^{\zeta}\right)$ and threedimensional basis functions $S_{\alpha}^{i} S_{\alpha}^{j} S_{\alpha}^{k}$ are enumerated so that the most frequently changing index is $\eta$, then $\xi$ and then $\zeta$ (and so as $j, i, k$ ). Then the blocks are of the form

$$
\begin{aligned}
L_{\alpha M^{\prime} M^{\prime}}= & -S_{z_{\alpha}} \otimes S_{x_{\alpha}} \otimes D_{y_{\alpha}}-S_{z_{\alpha}} \otimes D_{x_{\alpha}} \otimes S_{y_{\alpha}}+\left(J(J+1)-2 M^{\prime 2}\right) S_{z_{\alpha}} \otimes S_{x_{\alpha}} \otimes\left(Y_{\alpha r 2} S_{y_{\alpha}}\right) \\
& -D_{z_{\alpha}}^{M^{\prime}} \otimes S_{x_{\alpha}} \otimes\left(Y_{\alpha r 2} S_{y_{\alpha}}\right)-D_{z_{\alpha}}^{M^{\prime}} \otimes\left(X_{\alpha r 2} S_{x_{\alpha}}\right) \otimes S_{y_{\alpha}} \\
& +S_{z_{\alpha}} \otimes\left(V_{\alpha} S_{x_{\alpha}}\right) \otimes S_{y_{\alpha}}+S_{z_{\alpha}} \otimes S_{x_{\alpha}} \otimes\left(\widehat{V}_{\alpha} S_{y_{\alpha}}\right)-E^{*} S_{z_{\alpha}} \otimes S_{x_{\alpha}} \otimes S_{y_{\alpha}} \\
L_{\alpha M^{\prime} M^{\prime} \pm 1}= & \pm \widehat{\lambda}^{J, \pm M^{\prime}} D_{z_{\alpha}}^{M^{\prime} \pm} \otimes S_{x_{\alpha}} \otimes\left(Y_{\alpha r 2} S_{y_{\alpha}}\right) .
\end{aligned}
$$

Here we have introduced the "one-dimensional" matrices with elements

$$
\begin{aligned}
& \left(S_{z_{\alpha}}\right)_{\zeta k}=S_{\alpha}^{k}\left(z_{\alpha}^{\zeta}\right), \quad\left(S_{x_{\alpha}}\right)_{\xi i}=S_{\alpha}^{i}\left(x_{\alpha}^{\zeta}\right), \quad\left(S_{y_{\alpha}}\right)_{\eta j}=S_{\alpha}^{j}\left(y_{\alpha}^{\eta}\right), \\
& \left(D_{x_{\alpha}}\right)_{\xi_{i}}=\left(\frac{d^{2}}{d x_{\alpha}^{2}} S_{\alpha}^{i}\right)\left(x_{\alpha}^{\xi}\right), \quad\left(D_{y_{\alpha}}\right)_{\eta j}=\left(\frac{d^{2}}{d y_{\alpha}^{2}} S_{\alpha}^{j}\right)\left(y_{\alpha}^{\eta}\right), \\
& \left(D_{z_{\alpha}}^{M^{\prime}}\right)_{\zeta k}=\left(\boldsymbol{d}_{z_{\alpha}}^{M^{\prime}} S_{\alpha}^{k}\right)\left(z_{\alpha}^{\zeta}\right), \quad\left(D_{z_{\alpha}}^{M^{\prime} \pm}\right)_{\zeta k}=\left(\boldsymbol{d}_{z_{\alpha}}^{M^{\prime} \pm} S_{\alpha}^{k}\right)\left(z_{\alpha}^{\zeta}\right), \\
& \left(X_{\alpha r 2}\right)_{\xi_{i}}=\delta_{\xi_{i}} \frac{1}{\left(x_{\alpha}^{\xi}\right)^{2}}, \quad\left(Y_{\alpha r 2}\right)_{\eta j}=\delta_{\eta j} \frac{1}{\left(y_{\alpha}^{\eta}\right)^{2}} \text {, } \\
& \left(V_{\alpha}\right)_{\xi i}=\delta_{\xi i} V_{\alpha}\left(x_{\alpha}^{\xi}\right), \quad\left(\widehat{V}_{\alpha}\right)_{\eta j}=\delta_{\eta j} \widehat{V}_{\alpha}\left(y_{\alpha}^{\eta}\right),
\end{aligned}
$$

where we have used the notation

$$
\begin{aligned}
& \boldsymbol{d}_{z_{\alpha}}^{M^{\prime}}=\left(1-\left(z_{\alpha}\right)^{2}\right) \frac{d^{2}}{d z_{\alpha}^{2}}-2\left(M^{\prime}+1\right) z_{\alpha} \frac{d}{d z_{\alpha}}-M^{\prime}\left(M^{\prime}+1\right), \\
& \boldsymbol{d}_{z_{\alpha}}^{M^{\prime}+}=-\left(1-\left(z_{\alpha}\right)^{2}\right) \frac{d}{d z_{\alpha}}+2\left(M^{\prime}+1\right) z_{\alpha}, \quad \boldsymbol{d}_{z_{\alpha}}^{M^{\prime}-}=-\frac{d}{d z_{\alpha}} .
\end{aligned}
$$


Let us find the diagonal representation

$$
\left(\bar{W}_{z_{\alpha}}^{M^{\prime}}, W_{z_{\alpha}}^{M^{\prime}}, \widetilde{\Lambda}_{z_{\alpha}}^{M^{\prime}}\right)=\mathfrak{D}\left(D_{z_{\alpha}}^{M^{\prime}}, S_{z_{\alpha}}\right), \quad M^{\prime}=M_{0}, \cdots, J .
$$

We introduce block diagonal matrices

$$
\begin{aligned}
& \overline{\mathcal{W}}_{z_{\alpha}}=\operatorname{diag}\left\{\bar{W}_{z_{\alpha}}^{M_{0}} \otimes I \otimes I, \bar{W}_{z_{\alpha}}^{M_{0}+1} \otimes I \otimes I, \cdots, \bar{W}_{z_{\alpha}}^{J} \otimes I \otimes I\right\}, \\
& \mathcal{W}_{z_{\alpha}}=\operatorname{diag}\left\{W_{z_{\alpha}}^{M_{0}} \otimes I \otimes I, W_{z_{\alpha}}^{M_{0}+1} \otimes I \otimes I, \cdots, W_{z_{\alpha}}^{J} \otimes I \otimes I\right\} .
\end{aligned}
$$

Then the matrix $L_{\alpha}$ can be written in the form

$$
L_{\alpha}=\overline{\mathcal{W}}_{z_{\alpha}}^{-1} L_{\alpha}^{x y} \mathcal{W}_{z_{\alpha}}^{-1}
$$

where $L_{\alpha}^{x y}$ is a block tridiagonal matrix with blocks $L_{\alpha M^{\prime} M^{\prime}-1}^{x y}, L_{\alpha M^{\prime} M^{\prime}}^{x y}$ and $L_{\alpha M^{\prime} M^{\prime}+1}^{x y}$ in row $M^{\prime}=M_{0}, \cdots, J$,

$$
\begin{aligned}
L_{\alpha M^{\prime} M^{\prime}}^{x y}= & I \otimes\left(-S_{x_{\alpha}} \otimes D_{y_{\alpha}}-D_{x_{\alpha}} \otimes S_{y_{\alpha}}+\left(J(J+1)-2 M^{\prime 2}\right) S_{x_{\alpha}} \otimes\left(Y_{\alpha r 2} S_{y_{\alpha}}\right)\right. \\
& \left.+\left(V_{\alpha} S_{x_{\alpha}}\right) \otimes S_{y_{\alpha}}+S_{x_{\alpha}} \otimes\left(\widehat{V}_{\alpha} S_{y_{\alpha}}\right)-E^{*} S_{x_{\alpha}} \otimes S_{y_{\alpha}}\right) \\
& -\widetilde{\Lambda}_{z_{\alpha}}^{M^{\prime}} \otimes\left(S_{x_{\alpha}} \otimes\left(Y_{\alpha r 2} S_{y_{\alpha}}\right)+\left(X_{\alpha r 2} S_{x_{\alpha}}\right) \otimes S_{y_{\alpha}}\right), \\
L_{\alpha M^{\prime} M^{\prime} \pm 1}^{x y}= & \pm \widehat{\lambda}^{J, \pm M^{\prime}}\left(\bar{W}_{z_{\alpha}}^{M^{\prime}} D_{z_{\alpha}}^{M^{\prime} \pm} W_{z_{\alpha}}^{M^{\prime} \pm 1}\right) \otimes S_{x_{\alpha}} \otimes\left(Y_{\alpha r 2} S_{y_{\alpha}}\right) .
\end{aligned}
$$

Now in order to build the preconditioner we introduce the block diagonal matrix $\mathcal{L}=$ $\operatorname{diag}\left\{\mathcal{L}_{1}, \mathcal{L}_{2}, \mathcal{L}_{3}\right\}$. This matrix is the approximation of the matrix $L$ of the operator at the left-hand side of 3D FM Eqs. (3.8). Its inverse $\mathcal{L}^{-1}$ is used as a preconditioner of the matrix $H-E^{*} S$ in the eigenvalue problem (3.10). The block $\mathcal{L}_{\alpha}$ is given by

$$
\mathcal{L}_{\alpha} \equiv \overline{\mathcal{W}}_{z_{\alpha}}^{-1} \mathcal{L}_{\alpha}^{x y} \mathcal{W}_{z_{\alpha}}^{-1}
$$

where $\mathcal{L}_{\alpha}^{x y}$ has exactly the same block structure as the matrix $L_{\alpha}^{x y}$ with blocks $\mathcal{L}_{\alpha M^{\prime} M^{\prime}-1}^{x y} \approx$ $L_{\alpha M^{\prime} M^{\prime}-1}^{x y}, \mathcal{L}_{\alpha M^{\prime} M^{\prime}}^{x y} \approx L_{\alpha M^{\prime} M^{\prime}}^{x y}$ and $\mathcal{L}_{\alpha M^{\prime} M^{\prime}+1}^{x y} \approx L_{\alpha M^{\prime} M^{\prime}+1}^{x y}$ in row $M^{\prime}=M_{0}, \cdots, J$. To obtain these blocks we note that under some assumptions on the basis of functions $S_{\alpha}^{k}(z)$ and the choice of collocation points $z_{\alpha}^{\tau}$ given in Appendix A the following approximate equalities hold

$$
\begin{aligned}
& \widetilde{\Lambda}_{z_{\alpha}}^{M^{\prime}} \approx \Lambda_{z_{\alpha}}^{M^{\prime}} \\
& \quad \equiv \operatorname{diag}\left\{-M^{\prime}\left(M^{\prime}+1\right),-\left(M^{\prime}+1\right)\left(M^{\prime}+2\right), \cdots,-\left(M^{\prime}+n_{z_{\alpha}}-1\right)\left(M^{\prime}+n_{z_{\alpha}}\right)\right\}, \\
& \bar{W}_{z_{\alpha}}^{M^{\prime}} D_{z_{\alpha}}^{M^{\prime} \pm} W_{z_{\alpha}}^{M^{\prime} \pm 1} \approx \Lambda_{z_{\alpha}}^{M^{\prime} \pm},
\end{aligned}
$$

where

$$
\Lambda_{z_{\alpha}}^{M^{\prime}+}=\left(\begin{array}{ccccc}
0 & & & & \\
-\lambda^{M^{\prime}+1, M^{\prime}} & 0 & & & \\
& -\lambda^{M^{\prime}+2, M^{\prime}} & 0 & & \\
& & & \ddots & \\
& & & -\lambda^{M^{\prime}+n_{z_{\alpha}}-1, M^{\prime}} & 0
\end{array}\right) \text {, }
$$




$$
\Lambda_{z_{\alpha}}^{M^{\prime}-}=\left(\begin{array}{ccccc}
0 & \lambda^{M^{\prime},-M^{\prime}} & & & \\
& 0 & \lambda^{M^{\prime}+1,-M^{\prime}} & & \\
& & \ddots & & \\
& & & 0 & \lambda^{M^{\prime}+n_{z_{\alpha}}-2,-M^{\prime}} \\
& & & & 0
\end{array}\right) .
$$

The justification of approximate equalities (3.31) is given in Appendix A. Then the expressions for matrices $\mathcal{L}_{\alpha M^{\prime} M^{\prime}-1}^{x y}, \mathcal{L}_{\alpha M^{\prime} M^{\prime}}^{x y}, \mathcal{L}_{\alpha M^{\prime} M^{\prime}+1}^{x y}$ are obtained from (3.28), (3.29) by making substitutions $\widetilde{\Lambda}_{z_{\alpha}}^{M^{\prime}} \rightarrow \Lambda_{z_{\alpha}}^{M^{\prime}}, \bar{W}_{z_{\alpha}}^{M^{\prime}} D_{z_{\alpha}}^{M^{\prime} \pm} W_{z_{\alpha}}^{M^{\prime} \pm 1} \rightarrow \Lambda_{z_{\alpha}}^{M^{\prime} \pm}$. They are

$$
\begin{aligned}
\mathcal{L}_{\alpha M^{\prime} M^{\prime}}^{x y}= & I \otimes\left(-S_{x_{\alpha}} \otimes D_{y_{\alpha}}-D_{x_{\alpha}} \otimes S_{y_{\alpha}}+\left(J(J+1)-2 M^{\prime 2}\right) S_{x_{\alpha}} \otimes\left(Y_{\alpha r 2} S_{y_{\alpha}}\right)\right. \\
& \left.+\left(V_{\alpha} S_{x_{\alpha}}\right) \otimes S_{y_{\alpha}}+S_{x_{\alpha}} \otimes\left(\widehat{V}_{\alpha} S_{y_{\alpha}}\right)-E^{*} S_{x_{\alpha}} \otimes S_{y_{\alpha}}\right) \\
& -\Lambda_{z_{\alpha}}^{M^{\prime}} \otimes\left(S_{x_{\alpha}} \otimes\left(Y_{\alpha r 2} S_{y_{\alpha}}\right)+\left(X_{\alpha r 2} S_{x_{\alpha}}\right) \otimes S_{y_{\alpha}}\right), \\
\mathcal{L}_{\alpha M^{\prime} M^{\prime} \pm 1}^{x y}= & \pm \widehat{\lambda}^{J, \pm M^{\prime}} \Lambda_{z_{\alpha}}^{M^{\prime} \pm} \otimes S_{x_{\alpha}} \otimes\left(Y_{\alpha r 2} S_{y_{\alpha}}\right) .
\end{aligned}
$$

By diagonalizing matrices related to operators in variable $z_{\alpha}$ we have done the first step of the MDA. In the remaining part of this subsection we deal with the approximate matrix $\mathcal{L}$ and show how to invert it efficiently by diagonalizing matrices related to operators in $x_{\alpha}$ and $y_{\alpha}$.

We proceed further by noting that each diagonal block $\mathcal{L}_{\alpha M^{\prime} M^{\prime}}^{x y}$ of the matrix $\mathcal{L}_{\alpha}^{x y}$ has a block diagonal structure with "two-dimensional" blocks $\mathcal{M}_{\alpha \ell}^{M^{\prime}}$ of the form

$$
\begin{aligned}
\mathcal{M}_{\alpha \ell}^{M^{\prime}}= & S_{x_{\alpha}} \otimes\left[-D_{y_{\alpha}}+\left(J(J+1)-2 M^{\prime 2}\right)\left(Y_{\alpha r 2} S_{y_{\alpha}}\right)\right. \\
& \left.+\ell(\ell+1)\left(Y_{\alpha r 2} S_{y_{\alpha}}\right)+\left(\widehat{V}_{\alpha} S_{y_{\alpha}}\right)-E^{*} S_{y_{\alpha}}\right] \\
& +\left[-D_{x_{\alpha}}+\ell(\ell+1)\left(X_{\alpha r 2} S_{x_{\alpha}}\right)+\left(V_{\alpha} S_{x_{\alpha}}\right)\right] \otimes S_{y_{\alpha}}, \quad \ell=M^{\prime}, \cdots, M^{\prime}+n_{z_{\alpha}}-1 .
\end{aligned}
$$

Likewise, the off-diagonal blocks $\mathcal{L}_{\alpha M^{\prime} M^{\prime} \pm 1}^{x y}$ are block matrices with nontrivial blocks $\mathcal{M}_{\alpha \ell}^{M^{\prime} \pm}$ on subdiagonal of $\mathcal{L}_{\alpha M^{\prime} M^{\prime}+1}^{x y}$ and superdiagonal of $\mathcal{L}_{\alpha M^{\prime} M^{\prime}-1}^{x y}$. They have the form

$$
\begin{aligned}
& \mathcal{M}_{\alpha \ell}^{M^{\prime}+}=\widehat{\lambda}^{J, M^{\prime}}\left(\Lambda_{z_{\alpha}}^{M^{\prime}+}\right)_{\ell-M^{\prime}+1, \ell-M^{\prime}} S_{x_{\alpha}} \otimes\left(Y_{\alpha r 2} S_{y_{\alpha}}\right), \quad \ell=M^{\prime}+1, \cdots, M^{\prime}+n_{z_{\alpha}}-1, \\
& \mathcal{M}_{\alpha \ell}^{M^{\prime}-}=-\widehat{\lambda}^{J,-M^{\prime}}\left(\Lambda_{z_{\alpha}}^{M^{\prime}-}\right)_{\ell-M^{\prime}+1, \ell-M^{\prime}+2} S_{x_{\alpha}} \otimes\left(Y_{\alpha r 2} S_{y_{\alpha}}\right), \quad \ell=M^{\prime}, \cdots, M^{\prime}+n_{z_{\alpha}}-2 .
\end{aligned}
$$

Thus the matrix $\mathcal{L}_{\alpha}^{x y}$ has the form shown in Fig. 3 with blocks with indices ${ }_{\ell}^{M^{\prime}}$ and ${ }_{\ell}^{M^{\prime} \pm}$ denoting matrices $\mathcal{M}_{\alpha \ell}^{M^{\prime}}, \mathcal{M}_{\alpha \ell}^{M^{\prime} \pm}$. Now let

$$
\left(\bar{W}_{x_{\alpha}}^{\ell}, W_{x_{\alpha}}^{\ell}, \Lambda_{x_{\alpha}}^{\ell}\right)=\mathfrak{D}\left(-D_{x_{\alpha}}+\ell(\ell+1) X_{\alpha r 2} S_{x_{\alpha}}+V_{\alpha} S_{x_{\alpha}}, S_{x_{\alpha}}\right), \quad \ell=M_{0}, \cdots, J+n_{z_{\alpha}}-1 .
$$



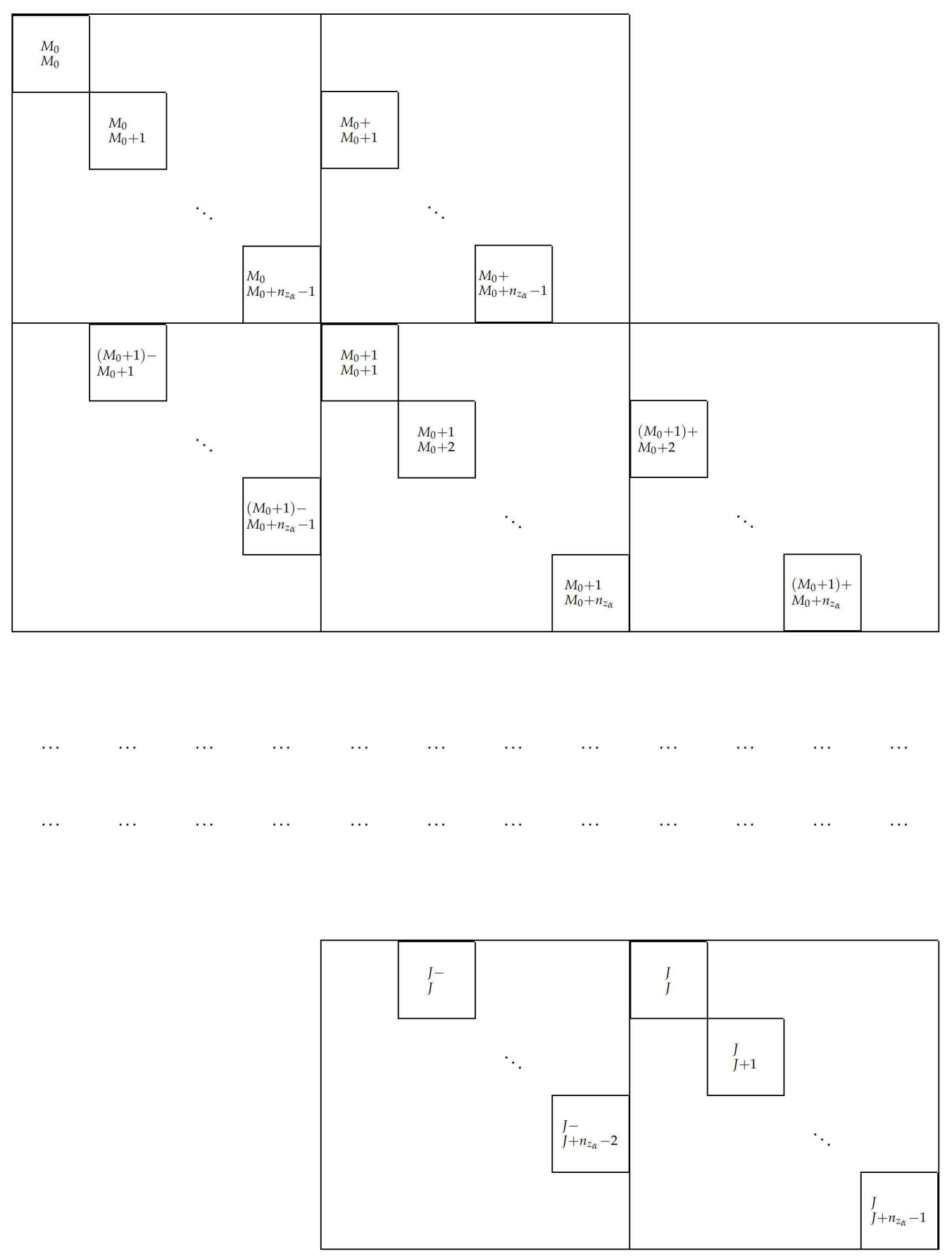

Figure 3: Structure of the matrices $\mathcal{L}_{\alpha}^{x y}, \mathcal{L}_{\alpha}^{y}$ and $\mathcal{L}_{\alpha}^{0}$. 
Introduce new block diagonal matrices

$$
\begin{aligned}
& \overline{\mathcal{W}}_{x_{\alpha}}=\operatorname{diag}\left\{\overline{\mathcal{W}}_{x_{\alpha}}^{M_{0}}, \ldots, \overline{\mathcal{W}}_{x_{\alpha}}^{M_{0}+n_{z_{\alpha}}-1}, \overline{\mathcal{W}}_{x_{\alpha}}^{M_{0}+1}, \ldots, \overline{\mathcal{W}}_{x_{\alpha}}^{M_{0}+n_{z_{\alpha}}}, \ldots \ldots ., \overline{\mathcal{W}}_{x_{\alpha}}^{J}, \ldots, \overline{\mathcal{W}}_{x_{\alpha}}^{J+n_{z_{\alpha}}-1}\right\}, \\
& \mathcal{W}_{x_{\alpha}}=\operatorname{diag}\left\{\mathcal{W}_{x_{\alpha}}^{M_{0}}, \cdots, \mathcal{W}_{x_{\alpha}}^{M_{0}+n_{z_{\alpha}}-1}, \mathcal{W}_{x_{\alpha}}^{M_{0}+1}, \cdots, \mathcal{W}_{x_{\alpha}}^{M_{0}+n_{z_{\alpha}}}, \ldots \cdots \cdots, \mathcal{W}_{x_{\alpha}}^{J}, \cdots, \mathcal{W}_{x_{\alpha}}^{J+n_{z_{\alpha}}-1}\right\},
\end{aligned}
$$

where $\overline{\mathcal{W}}_{x_{\alpha}}^{\ell}=\bar{W}_{x_{\alpha}}^{\ell} \otimes I$ and $\mathcal{W}_{x_{\alpha}}^{\ell}=W_{x_{\alpha}}^{\ell} \otimes I$. Then one can check that

$$
\mathcal{L}_{\alpha}^{x y}=\overline{\mathcal{W}}_{x_{\alpha}}^{-1} \mathcal{L}_{\alpha}^{y} \mathcal{W}_{x_{\alpha}}^{-1}
$$

where the new matrix $\mathcal{L}_{\alpha}^{y}$ has exactly the same form as $\mathcal{L}_{\alpha}^{x y}$ shown in Fig. 3 with blocks $\mathcal{N}_{\alpha \ell}^{M^{\prime}}, \mathcal{N}_{\alpha \ell}^{M^{\prime} \pm}$. These new blocks are

$$
\begin{aligned}
& \mathcal{N}_{\alpha \ell}^{M^{\prime}}=I \otimes\left(-D_{y_{\alpha}}+\left(J(J+1)-2 M^{\prime 2}+\ell(\ell+1)\right)\left(Y_{\alpha r 2} S_{y_{\alpha}}\right)+\widehat{V}_{\alpha} S_{y_{\alpha}}-E^{*} S_{y_{\alpha}}\right)+\Lambda_{x_{\alpha}}^{\ell} \otimes S_{y_{\alpha}} \\
& \mathcal{N}_{\alpha \ell}^{M^{\prime}+}=\widehat{\lambda}^{J, M^{\prime}}\left(\Lambda_{z_{\alpha}}^{M^{\prime}+}\right)_{\ell-M^{\prime}+1, \ell-M^{\prime}} I \otimes\left(Y_{\alpha r 2} S_{y_{\alpha}}\right), \quad \ell=M^{\prime}+1, \cdots, M^{\prime}+n_{z_{\alpha}}-1 \\
& \mathcal{N}_{\alpha \ell}^{M^{\prime}-}=-\widehat{\lambda}^{J,-M^{\prime}}\left(\Lambda_{z_{\alpha}}^{M^{\prime}-}\right)_{\ell-M^{\prime}+1, \ell-M^{\prime}+2} I \otimes\left(Y_{\alpha r 2} S_{y_{\alpha}}\right), \quad \ell=M^{\prime}, \cdots, M^{\prime}+n_{z_{\alpha}}-2
\end{aligned}
$$

They are in turn block diagonal

$$
\begin{aligned}
& \mathcal{N}_{\alpha \ell}^{M^{\prime}}=\operatorname{diag}\left\{\mathcal{N}_{\alpha \ell 1}^{M^{\prime}}, \mathcal{N}_{\alpha \ell 2}^{M^{\prime}}, \cdots, \mathcal{N}_{\alpha \ell n_{x_{\alpha}}}^{M^{\prime}}\right\} \\
& \mathcal{N}_{\alpha \ell}^{M^{\prime} \pm}=\operatorname{diag}\left\{\mathcal{N}_{\alpha \ell 0}^{M^{\prime} \pm}, \mathcal{N}_{\alpha \ell 0}^{M^{\prime} \pm}, \cdots, \mathcal{N}_{\alpha \ell 0}^{M^{\prime} \pm}\right\}
\end{aligned}
$$

where the "one-dimensional" matrices have the form

$$
\begin{aligned}
& \mathcal{N}_{\alpha \ell i_{x_{\alpha}}}^{M^{\prime}}=-D_{y_{\alpha}}+\widehat{V}_{\alpha} S_{y_{\alpha}}-E^{*} S_{y_{\alpha}}+\left(\Lambda_{x_{\alpha}}^{\ell}\right)_{i_{x_{\alpha}}, i_{x_{\alpha}}} S_{y_{\alpha}}+\left(J(J+1)-2 M^{\prime 2}+\ell(\ell+1)\right)\left(Y_{\alpha r 2} S_{y_{\alpha}}\right), \\
& \quad \ell=M^{\prime}, \cdots, M^{\prime}+n_{z_{\alpha}}-1, \quad i_{x_{\alpha}}=1, \cdots, n_{x_{\alpha}} \\
& \mathcal{N}_{\alpha \ell 0}^{M^{\prime}+}=\widehat{\lambda}^{J, M^{\prime}}\left(\Lambda_{z_{\alpha}}^{M^{\prime}+}\right)_{\ell-M^{\prime}+1, \ell-M^{\prime}}\left(Y_{\alpha r 2} S_{y_{\alpha}}\right), \quad \ell=M^{\prime}+1, \cdots, M^{\prime}+n_{z_{\alpha}}-1 \\
& \mathcal{N}_{\alpha \ell 0}^{M^{\prime}-}=-\widehat{\lambda}^{J,-M^{\prime}}\left(\Lambda_{z_{\alpha}}^{M^{\prime}-}\right)_{\ell-M^{\prime}+1, \ell-M^{\prime}+2}\left(Y_{\alpha r 2} S_{y_{\alpha}}\right), \quad \ell=M^{\prime}, \cdots, M^{\prime}+n_{z_{\alpha}}-2
\end{aligned}
$$

Now we make the last step of diagonalization process. Introduce

$$
\begin{gathered}
\left(\bar{W}_{y_{\alpha}}^{\ell i_{x_{\alpha}}}, W_{y_{\alpha}}^{\ell i_{x_{\alpha}}}, \Lambda_{y_{\alpha}}^{\ell i_{x_{\alpha}}}\right)= \\
\mathcal{D}\left(-D_{y_{\alpha}}+\widehat{V}_{\alpha} S_{y_{\alpha}}-E^{*} S_{y_{\alpha}}+\left(\Lambda_{x_{\alpha}}^{\ell}\right)_{i_{x_{\alpha}} i_{x_{\alpha}}} S_{y_{\alpha}}, Y_{\alpha r 2} S_{y_{\alpha}}\right), \\
\ell=M_{0}, \cdots, J+n_{z_{\alpha}}-1, \quad i_{x_{\alpha}}=1, \cdots, n_{x_{\alpha}} .
\end{gathered}
$$


Then using the matrices

$$
\begin{aligned}
& \overline{\mathcal{W}}_{y_{\alpha}}=\operatorname{diag}\left\{\overline{\mathcal{W}}_{y_{\alpha}}^{M_{0}}, \cdots, \overline{\mathcal{W}}_{y_{\alpha}}^{M_{0}+n_{z_{\alpha}}-1}, \overline{\mathcal{W}}_{y_{\alpha}}^{M_{0}+1}, \cdots, \overline{\mathcal{W}}_{y_{\alpha}}^{M_{0}+n_{z_{\alpha}}}, \cdots \cdots \cdots, \overline{\mathcal{W}}_{y_{\alpha}}^{J}, \cdots, \overline{\mathcal{W}}_{y_{\alpha}}^{I+n_{z_{\alpha}}-1}\right\}, \\
& \mathcal{W}_{y_{\alpha}}=\operatorname{diag}\left\{\mathcal{W}_{y_{\alpha}}^{M_{0}}, \cdots, \mathcal{W}_{y_{\alpha}}^{M_{0}+n_{z_{\alpha}}-1}, \mathcal{W}_{y_{\alpha}}^{M_{0}+1}, \cdots, \mathcal{W}_{y_{\alpha}}^{M_{0}+n_{z_{\alpha}}}, \cdots \cdots \cdots, \mathcal{W}_{y_{\alpha}}^{J}, \cdots, \mathcal{W}_{y_{\alpha}}^{J+n_{z_{\alpha}}-1}\right\},
\end{aligned}
$$

where $\overline{\mathcal{W}}_{y_{\alpha}}^{\ell}$ and $\mathcal{W}_{y_{\alpha}}^{\ell}$ are in turn block diagonal matrices of the form

$$
\begin{aligned}
& \overline{\mathcal{W}}_{y_{\alpha}}^{\ell}=\operatorname{diag}\left\{\bar{W}_{y_{\alpha}}^{\ell 1}, \ldots, \bar{W}_{y_{\alpha}}^{\ell n_{x_{\alpha}}}\right\}, \\
& \mathcal{W}_{y_{\alpha}}^{\ell}=\operatorname{diag}\left\{W_{y_{\alpha}}^{\ell 1}, \cdots, W_{y_{\alpha}}^{\ell n_{x_{\alpha}}}\right\},
\end{aligned}
$$

we represent

$$
\mathcal{L}_{\alpha}^{y}=\overline{\mathcal{W}}_{y_{\alpha}}^{-1} \mathcal{L}_{\alpha}^{0} \mathcal{W}_{y_{\alpha}}^{-1}
$$

Here the matrix $\mathcal{L}_{\alpha}^{0}$ has exactly the same form as $\mathcal{L}_{\alpha}^{x y}$ and $\mathcal{L}_{\alpha}^{y}$ shown in Fig. 3 with blocks $\mathcal{P}_{\alpha \ell}^{M^{\prime}}, \mathcal{P}_{\alpha \ell}^{M^{\prime} \pm}$. These new blocks are diagonal matrices with elements

$$
\begin{gathered}
\left(\mathcal{P}_{\alpha \ell}^{M^{\prime}}\right)_{\left(i_{x_{\alpha}}-1\right) n_{y \alpha}+i_{y_{\alpha}},\left(i_{x_{\alpha}}-1\right) n_{y_{\alpha}}+i_{y_{\alpha}}}=\left(\Lambda_{y_{\alpha}}^{\ell, i_{x_{\alpha}}}\right)_{i_{y_{\alpha}}, i_{y_{\alpha}}}+J(J+1)-2 M^{\prime 2}+\ell(\ell+1), \\
\left(\mathcal{P}_{\alpha \ell}^{M^{\prime}+}\right)_{\left(i_{x_{\alpha}}-1\right) n_{y_{\alpha}}+i_{y_{\alpha}}\left(i_{x_{\alpha}}-1\right) n_{y_{\alpha}}+i_{y_{\alpha}}}=\widehat{\lambda}^{J, M^{\prime}}\left(\Lambda_{z_{\alpha}}^{M^{\prime}+}\right)_{\ell-M^{\prime}+1, \ell-M^{\prime}} \\
\left(\mathcal{P}_{\alpha \ell}^{M^{\prime}-}\right)_{\left(i_{x_{\alpha}}-1\right) n_{y_{\alpha}}+i_{y_{\alpha}}\left(i_{x_{\alpha}}-1\right) n_{y_{\alpha}}+i_{y_{\alpha}}}=-\widehat{\lambda}^{J,-M^{\prime}}\left(\Lambda_{z_{\alpha}}^{M^{\prime}-}\right)_{\ell-M^{\prime}+1, \ell-M^{\prime}+2^{\prime}} \\
i_{x_{\alpha}}=1, \cdots, n_{x_{\alpha}}, \quad i_{y_{\alpha}}=1, \cdots, n_{y_{\alpha}} .
\end{gathered}
$$

In other words the matrix $\mathcal{L}_{\alpha}^{0}$ is a band matrix with three nonzero diagonals. It is easy to show that there exists a permutation matrix $P$ such that

$$
\mathcal{L}_{\alpha}^{0}=P\left(P \mathcal{L}_{\alpha}^{0} P\right) P=P \widetilde{\mathcal{L}}_{\alpha}^{0} P,
$$

where $\widetilde{\mathcal{L}}_{\alpha}^{0}$ is block diagonal with each block being a three-diagonal matrix. Every block comprises rows of $\mathcal{L}_{\alpha}^{0}$ with numbers forming sets of numbers with equal remainders of the division by $\left(n_{z_{\alpha}}-1\right) n_{x_{\alpha}} n_{y_{\alpha}}$. Thus the matrix $\mathcal{L}_{\alpha}^{0}$ can be inverted fast.

Summarizing, we have obtained

$$
\mathcal{L}_{\alpha}=\overline{\mathcal{W}}_{z_{\alpha}}^{-1} \overline{\mathcal{W}}_{x_{\alpha}}^{-1} \overline{\mathcal{W}}_{y_{\alpha}}^{-1} P \widetilde{\mathcal{L}}_{\alpha}^{0} P \mathcal{W}_{y_{\alpha}}^{-1} \mathcal{W}_{x_{\alpha}}^{-1} \mathcal{W}_{z_{\alpha}}^{-1}
$$

and the approximate preconditioner can be presented in the form

$$
\mathcal{L}^{-1}=\operatorname{diag}\left\{\mathcal{L}_{1}^{-1}, \mathcal{L}_{2}^{-1}, \mathcal{L}_{3}^{-1}\right\},
$$


with

$$
\mathcal{L}_{\alpha}^{-1}=\mathcal{W}_{z_{\alpha}} \mathcal{W}_{x_{\alpha}} \mathcal{W}_{y_{\alpha}} P\left(\widetilde{\mathcal{L}}_{\alpha}^{0}\right)^{-1} P \overline{\mathcal{W}}_{y_{\alpha}} \overline{\mathcal{W}}_{x_{\alpha}} \overline{\mathcal{W}}_{z_{\alpha}}
$$

Formulae (3.51), (3.52) are the analogue of formula (3.20) and thus we have done the last step of the MDA applied to the matrix $\mathcal{L}$. The operations (3.44) are the most computer time and memory consuming operations in calculating and storing the preconditioner. It is needed to store and calculate the total amount of $\sum_{\alpha}\left(n_{M}+n_{z_{\alpha}}-1\right) n_{x_{\alpha}}$ pairs $\left(\bar{W}_{y_{\alpha}}^{\ell, i_{x_{\alpha}}}, W_{y_{\alpha}}^{\ell, i_{x_{\alpha}}}\right)$ of matrices of general form. Thus the estimated memory requirements are $2 \sum_{\alpha}\left(n_{z_{\alpha}}+n_{M}\right) n_{x_{\alpha}} n_{y_{\alpha}}^{2}$ numbers and the computational cost scales as $\mathcal{O}\left(\sum_{\alpha}\left(n_{z_{\alpha}}+\right.\right.$ $\left.\left.n_{M}\right) n_{x_{\alpha}} n_{y_{\alpha}}^{3}\right)$. Finally, each matrix-vector product with the preconditioner $\mathcal{L}^{-1}$ requires $\mathcal{O}\left(n_{M} \sum_{\alpha} n_{x_{\alpha}} n_{z_{\alpha}} n_{y_{\alpha}}\left(n_{x_{\alpha}}+n_{z_{\alpha}}+n_{y_{\alpha}}\right)\right)$ operations.

\subsection{Matrix storage}

In the last part of this section we discuss the storage of the full matrix $H$ which is the most memory consuming part of the scheme. In this discussion it is more convenient to refer to the 3D FM equations written in the form (3.3). Let $H=\widetilde{L}+\widetilde{R}$ with $\widetilde{L}$ and $\widetilde{R}$ being the discretized versions of the operators at the left- and right-hand sides of 3D FM Eqs. (3.3). The matrices $\widetilde{L}$ and $\widetilde{R}$ contain diagonal and off-diagonal with respect to component numbers $\alpha$ blocks of the full matrix $H$ and thus can be stored independently. Now one obvious way is to keep those matrices in memory as sparse matrices using one of the well known storage schemes like the CSR format [46]. The estimated storage size and computational cost of matrix-vector product are $\left(3 n_{M}-2\right) r^{3} \sum_{\alpha} n_{x_{\alpha}} n_{y_{\alpha}} n_{z_{\alpha}}$ numbers and multiplications for $\widetilde{L}$ and $2 n_{M}^{2} r^{3} \sum_{\alpha} n_{x_{\alpha}} n_{y_{\alpha}} n_{z_{\alpha}}$ for $\widetilde{R}$. Here $r$ is the overlap rate introduced in Subsection 3.1.

These requirements can be drastically lowered in the case of using the same basis set for expanding components $\widehat{\psi}_{\alpha M M^{\prime}}^{\tau \tau}$ with different $M^{\prime}$ and identical sets of collocation points for Eqs. (3.8) with different $M^{\prime}$. One possible storage scheme that we use in the calculations is presented here. The blocks $\widetilde{L}_{\alpha}$ of the matrix $\widetilde{L}$ are stored in the form

$$
\widetilde{L}_{\alpha}=I_{M} \otimes D_{x_{\alpha} y_{\alpha} z_{\alpha}}+M S_{z_{\alpha}} \otimes S Y S_{x_{\alpha} y_{\alpha}}+M D_{z_{\alpha}} \otimes X S Y S_{x_{\alpha} y_{\alpha}} .
$$

Here the matrix $I_{M}$ is the $n_{M} \times n_{M}$ identity matrix in the space of momenta numbers $M^{\prime}$. The matrices that are stored in memory as sparse matrices in CSR format are: $D_{x_{\alpha} y_{\alpha} z_{\alpha}}$ is the discretized version of the three-dimensional operator

$$
-\frac{\partial^{2}}{\partial y_{\alpha}^{2}}-\frac{\partial^{2}}{\partial x_{\alpha}^{2}}-\left(\frac{1}{y_{\alpha}^{2}}+\frac{1}{x_{\alpha}^{2}}\right)\left(1-z_{\alpha}^{2}\right) \frac{\partial^{2}}{\partial z_{\alpha}^{2}}+V_{\alpha}\left(x_{\alpha}\right)+\sum_{\beta \neq \alpha} V_{\beta}^{(1)}\left(x_{\beta}\left(X_{\alpha}\right), y_{\beta}\left(X_{\alpha}\right)\right)-E
$$


and other matrices have the form

$$
\begin{aligned}
& S Y S_{x_{\alpha} y_{\alpha}}=S_{x_{\alpha}} \otimes\left(Y_{\alpha r 2} S_{y_{\alpha}}\right) \\
& X S Y S_{x_{\alpha} y_{\alpha}}=\left(X_{\alpha r 2} S_{x_{\alpha}}\right) \otimes S_{y_{\alpha}}+S_{x_{\alpha}} \otimes\left(Y_{\alpha r 2} S_{y_{\alpha}}\right) \\
& M S_{z_{\alpha}}=J M \otimes S_{z_{\alpha}}+\Lambda_{M}^{-} \otimes D_{1 z_{\alpha}}-\Lambda_{M}^{+} \otimes\left(\left(I-Z_{\alpha}^{2}\right) D_{1 z_{\alpha}}\right)+2\left(\left(M M+I_{M}\right) \Lambda_{M}^{+}\right) \otimes\left(Z_{\alpha} S_{z_{\alpha}}\right) \\
& M D_{z_{\alpha}}=2\left(M M+I_{M}\right) \otimes\left(Z_{\alpha} D_{1 z_{\alpha}}\right)+\left(M M\left(M M+I_{M}\right)\right) \otimes S_{z_{\alpha}}
\end{aligned}
$$

Here we have additionally denoted by $D_{1 z_{\alpha}}$ and $Z_{\alpha}$ the matrices with elements

$$
\left(D_{1 z_{\alpha}}\right)_{\zeta k}=\left(\frac{d}{d z_{\alpha}} S_{\alpha}^{k}\right)\left(z_{\alpha}^{\zeta}\right), \quad\left(Z_{\alpha}\right)_{\zeta k}=\delta_{\zeta k} z_{\alpha}^{\zeta}
$$

and the matrices in the space of momenta numbers are

$$
\begin{aligned}
& J M=\operatorname{diag}\left\{J(J+1)-2 M_{0}^{2}, J(J+1)-2\left(M_{0}+1\right)^{2}, \cdots, J(J+1)-2 J^{2}\right\}, \\
& M M=\operatorname{diag}\left\{M_{0}, M_{0}+1, \cdots, J\right\} \text {, } \\
& \Lambda_{M}^{-}=\left(\begin{array}{ccccc}
0 & & & & \\
\hat{\lambda}^{J,-\left(M_{0}+1\right)} & 0 & & & \\
& \widehat{\lambda}^{J,-\left(M_{0}+2\right)} & 0 & & \\
& & \ddots & \\
& & \hat{\lambda}^{J,-J} & 0
\end{array}\right) \text {, } \\
& \Lambda_{M}^{+}=\left(\begin{array}{ccccc}
0 & \hat{\lambda}^{J, M_{0}} & & & \\
& 0 & \widehat{\lambda}^{J, M_{0}+1} & & \\
& & \ddots & & \\
& & & 0 & \widehat{\lambda}^{J, J-1} \\
& & & & 0
\end{array}\right) .
\end{aligned}
$$

If blocks of the matrix $\widetilde{L}$ are stored in the form (3.53), then it requires a storage of $\mathcal{O}\left(r^{3} \sum_{\alpha} n_{x_{\alpha}} n_{y_{\alpha}} n_{z_{\alpha}}+4 n_{M} r \sum_{\alpha} n_{z_{\alpha}}+2 r^{2} \sum_{\alpha} n_{x_{\alpha}} n_{y_{\alpha}}\right)$ numbers and it takes $\mathcal{O}\left(n_{M}\left(r^{3}+2 r^{2}+\right.\right.$ $\left.4 r) \sum_{\alpha} n_{x_{\alpha}} n_{y_{\alpha}} n_{z_{\alpha}}\right)$ multiplication operations for matrix-vector product.

The matrix $\widetilde{R}$ is a block matrix with blocks $\widetilde{R}_{\alpha \beta}$. If no permutational symmetry is taken into account, it has trivial diagonal blocks $\widetilde{R}_{\alpha \beta}=0, \alpha=\beta$. The nonzero blocks $\widetilde{R}_{\alpha \beta}$ can be stored in the form

$$
\widetilde{R}_{\alpha \beta}=F_{\alpha \beta} \widetilde{S}_{\alpha \beta}
$$

with

$$
\widetilde{S}_{\alpha \beta}=I_{M} \otimes S_{\alpha \beta},
$$

where the "three-dimensional" matrix $S_{\alpha \beta}$ that is stored in memory as CSR sparse matrix has elements

$$
\left(S_{\alpha \beta}\right)_{\zeta \xi \eta, k i j}=S_{\beta}^{k}\left(z_{\beta}\left(x_{\alpha}^{\xi}, y_{\alpha}^{\eta}, z_{\alpha}^{\zeta}\right)\right) S_{\beta}^{i}\left(x_{\beta}\left(x_{\alpha}^{\xi}, y_{\alpha}^{\eta}, z_{\alpha}^{\zeta}\right)\right) S_{\beta}^{j}\left(y_{\beta}\left(x_{\alpha}^{\zeta}, y_{\alpha}^{\eta}, z_{\alpha}^{\zeta}\right)\right) .
$$


Note that if different basis sets are used for expanding components $\widehat{\psi}_{\alpha M M^{\prime}}^{I \tau}$, the decomposition (3.58) still makes sense, but $\widetilde{S}_{\alpha \beta}$ cannot be presented in the form (3.59). Instead, it is then block diagonal with $n_{M}$ different blocks. Each stored in memory matrix $F_{\alpha \beta}$ is a block matrix with blocks $F_{\alpha \beta}^{M^{\prime} M^{\prime \prime}}, M^{\prime}, M^{\prime \prime}=M_{0}, \cdots, J$. Each block is a "three-dimensional" diagonal matrix with elements

$$
\begin{aligned}
\left(F_{\alpha \beta}^{M^{\prime} M^{\prime \prime}}\right)_{\zeta \xi \eta, k i j}= & -\delta_{\zeta k} \delta_{\xi i} \delta_{\eta j} \frac{x_{\alpha}^{\xi} y_{\alpha}^{\eta}}{x_{\beta}\left(x_{\alpha}^{\xi}, y_{\alpha}^{\eta}, z_{\alpha}^{\zeta}\right) y_{\beta}\left(x_{\alpha}^{\xi}, y_{\alpha}^{\eta}, z_{\alpha}^{\zeta}\right)} \frac{\left(1-\left(z_{\beta}\left(x_{\alpha}^{\xi}, y_{\alpha}^{\eta}, z_{\alpha}^{\zeta}\right)\right)^{2}\right)^{M^{\prime \prime} / 2}}{\left(1-\left(z_{\alpha}\right)^{2}\right)^{M^{\prime} / 2}} \\
& \times V_{\alpha}^{(\mathrm{s})}\left(x_{\alpha}^{\xi}, y_{\alpha}^{\eta}\right)(-1)^{M^{\prime \prime}-M^{\prime}} \frac{2}{\sqrt{2+2 \delta_{M^{\prime \prime} 0}}} F_{M^{\prime \prime} M^{\prime}}^{J \tau}\left(0, w_{\beta \alpha}, 0\right) .
\end{aligned}
$$

The matrix $F_{\alpha \beta}$ is stored in memory as a set of diagonal matrices. Storing blocks of matrix $\widetilde{R}$ in the form (3.58) requires storage of $\mathcal{O}\left(\left(n_{M}^{2}+r^{3}\right) \sum_{\alpha} n_{x_{\alpha}} n_{y_{\alpha}} n_{z_{\alpha}}\right)$ numbers and the same number of multiplication operations for matrix-vector product. This is the drastical progress as compared with storing $\widetilde{R}$ as sparse matrix of general form. However, simple analysis shows that using the forms (3.53) and (3.58) gives advantage compared with the full matrix CSR storage only if $n_{M}$ is greater than one.

\subsection{Discussion}

We notice that the presented in Subsections 3.2 and 3.3 tensor product structure of the preconditioner and the matrix $H$ is not specific to the collocation method. Rather, it is more a consequence of the choice of a tensor product basis, i.e. a basis consisting of three-dimensional basis functions being products of one-dimensional ones, and a special structure of the operator of the 3D FM equations (3.8). It can be shown that, for example, the Finite Element Method with appropriately chosen basis results in the same tensor product structure of the preconditioning matrix (3.51), (3.52) and the representation (3.53) of the matrix of the operator at the left-hand side of the 3D FM equations. However, in this case the properties of the matrix of the operator at the right-hand side of the 3D FM equations change. Particularly, it is not that easy to guarantee the good sparsity properties of this matrix due to the complicated overlap of the finite elements in different Jacobi coordinates $X_{\alpha}$ and $X_{\beta}$ (used to represent partial components with different indices $\widehat{\psi}_{\alpha M M^{\prime}}^{J \tau}\left(X_{\alpha}\right)$ and $\left.\widehat{\psi}_{\beta M M^{\prime}}^{J \tau}\left(X_{\beta}\right)\right)$.

In the calculations presented in the next section we use the method of spline collocation at Gauss points. This is a frequently used method for solving the elliptic PDEs that gives good convergence in many cases [47]. As is mentioned in the beginning of this section the theoretical convergence analysis of this method applied to the 3D FM equations is not between the aims of this work. It is a complicated task and requires a separate research to accomplish. A review of existing results on the convergence of spline collocation at Gauss points applied to two-dimensional elliptic PDEs can be found in [47]. 
However, our usage of the method presented in the next section (see also [33]) shows that a fast convergence is achieved.

\section{Examples}

In our realization of the described method the basis functions are in the space of quintic Hermite splines $S_{5}^{3}$ (splines of degree 5 with 2 continuous derivatives). Each basis function is local and nonzero only on two adjoining intervals of the grid. There are three functions associated with each grid node and thus for this choice of basis the defined in previous section overlap rate $r=6$. The spline nodes on the solution interval are chosen by mapping the equidistant grid with some function. The mapping function [48]

$$
\sin \left(z_{\alpha}(i) \pi / 2\right),
$$

where $z_{\alpha}(i)$ are points of the equidistant grid on interval $[-1,1]$, is used for splines in coordinate $z_{\alpha}$ in all calculations except those with high values of total orbital momentum $J$ presented in Table 3. In this case the more suited equidistant nodes have been used. For splines in coordinates $x_{\alpha}$ and $y_{\alpha}$ the mapping functions are chosen depending on the physical problem being solved. We use collocation at Gaussian points $[47,49]$. The largest modulus eigenvalues of the eigenvalue problem (3.10) are obtained by Implicitly Restarted Arnoldi Method (IRAM) [50,51]. We use the algorithm implemented in ARPACK [52] realization of IRAM. In the process of IRAM iterations the matrix $\left(H-E^{*} S\right)$ of (3.10) is being inverted by preconditioned GMRES algorithm [46]. Complex arithmetic is used in the calculations. The realization is written in $\mathrm{C}++$ programming language with the use of Intel Parallel Studio XE 2019 Update 4 software package. Parallelization of the program is achieved by using functions from Intel MKL [53] mathematical library. All calculations are done on a 6 core machine with Intel Xeon X5675 processor with 32 Gbytes RAM. In the presentation of results we adapt the notation

$$
\left(\alpha: n_{x_{\alpha}}, n_{y_{\alpha}}, n_{z_{\alpha}} ; \beta: n_{x_{\beta}}, n_{y_{\beta}}, n_{z_{\beta}}\right), \quad\left(\alpha: R_{x_{\alpha}}, R_{y_{\alpha}} ; \beta: R_{x_{\beta}}, R_{y_{\beta}}\right)
$$

for the basis sizes used to represent the partial components $\widehat{\psi}_{\alpha M M^{\prime}}^{I \tau}$ and lengths of intervals they are defined on.

We used two physical systems for the test calculations of bound state energies: the Helium atom and the molecular Helium trimer. All calculations for the Helium atom were conducted in atomic units. Since we have taken the results of [54] as benchmark results for atomic Helium energies, infinite ( $10^{15}$ a.u. in practice) Helium core mass was used in the calculations as in [54]. To generate nodes of spline in variable $x_{\alpha}\left(y_{\alpha}\right)$ on interval $\left[0, R_{x_{\alpha}}\right]\left(\left[0, R_{y_{\alpha}}\right]\right)$ we employed the mapping [55]

$$
4\left(\left(1+\frac{R_{x_{\alpha}}\left(R_{y_{\alpha}}\right)}{4}\right)^{u(i)}-1\right),
$$


Table 1: Comparison of the tensor product preconditioner approach and the Pardiso-based approach. Two lower energy levels $2^{1} P$ and $3^{1} P$ (i.e. $J=1, \tau=1$ states with the parity with respect to exchange of electrons $p=1$ ) of the Helium atom have been requested in each run. The solution domains are $(1: 36.0,36.0 ; 3: 9.0,6.0)$ a.u. The benchmark calculations [54] energy values are -2.12384308649810135925 and -2.05514636209194353689 a.u.

\begin{tabular}{|c|c|c|c|c|c|c|c|}
\hline \multirow[b]{2}{*}{ Basis sizes } & \multicolumn{3}{|c|}{ Tensor product preconditioner } & \multicolumn{3}{|l|}{ Pardiso } & \multirow[b]{2}{*}{$E$, a.u. } \\
\hline & $\begin{array}{l}\text { Total } \\
\text { memory / } \\
H-E^{*} S / \\
\text { Preconditioner } \\
\text { size, GB }\end{array}$ & $\begin{array}{l}\text { Calculate } \\
\text { matrices/ } \\
\text { IRAM } \\
\text { wall } \\
\text { time, } \\
\text { minutes }\end{array}$ & $\begin{array}{l}\text { Total } \\
\text { CPU } \\
\text { time, } \\
\text { minutes }\end{array}$ & $\begin{array}{l}\text { Total } \\
\text { memory / } \\
H-E^{*} S \\
\text { size, GB }\end{array}$ & $\begin{array}{l}\text { Calculate } \\
\text { matrices/ } \\
\text { IRAM } \\
\text { wall time, } \\
\text { minutes }\end{array}$ & $\begin{array}{l}\text { Total } \\
\text { CPU } \\
\text { time, } \\
\text { minutes }\end{array}$ & \\
\hline $\begin{array}{l}(1: 22,22,9 ; \\
3: 19,19,12)\end{array}$ & $0.14 / 0.09 / 0.01$ & $0.01 / 0.3$ & 2.1 & $2.29 / 0.28$ & $0.05 / 0.6$ & 2.7 & $\begin{array}{l}-2.12524 \\
-2.05654\end{array}$ \\
\hline $\begin{array}{l}\text { (1: 31, 31, 9; } \\
3: 22,22,15)\end{array}$ & $0.25 / 0.16 / 0.02$ & $0.02 / 0.7$ & 4.5 & $5.31 / 0.55$ & $0.04 / 1.9$ & 9.5 & $\begin{array}{l}-2.12365 \\
-2.05495\end{array}$ \\
\hline $\begin{array}{l}(1: 40,40,12 ; \\
3: 25,25,18)\end{array}$ & $0.45 / 0.30 / 0.04$ & $0.05 / 1.2$ & 7.3 & $12.5 / 1.07$ & $0.1 / 6.9$ & 35 & $\begin{array}{l}-2.12378 \\
-2.05508\end{array}$ \\
\hline $\begin{array}{l}(1: 49,49,12 ; \\
3: 28,28,21)\end{array}$ & $0.68 / 0.45 / 0.06$ & $0.08 / 1.9$ & 12 & $21.3 / 1.62$ & $0.1 / 14$ & 73 & $\begin{array}{l}-2.12385 \\
-2.05513\end{array}$ \\
\hline $\begin{array}{l}(1: 58,58,12 ; \\
3: 28,28,21)\end{array}$ & $0.85 / 0.56 / 0.10$ & $0.1 / 2.3$ & 15 & $29.4 / 2.04$ & $0.2 / 22$ & 120 & $\begin{array}{l}-2.12384 \\
-2.05514\end{array}$ \\
\hline
\end{tabular}

with $u(i)=x_{\alpha}(i) / R_{x_{\alpha}}\left(y_{\alpha}(i) / R_{y_{\alpha}}\right)$ and equidistant points $x_{\alpha}(i)\left(y_{\alpha}(i)\right)$. Splitting of the Coulomb potentials were done with the Merkuriev cut-off function of the form (2.6) with $v_{\alpha}=2.01, x_{0 \alpha}=1$ a.u. and $y_{0 \alpha}=+\infty$. The potentials $\widehat{V}_{\alpha}$ in Eqs. (3.8) are chosen to be

$$
\widehat{V}_{\alpha}\left(y_{\alpha}\right)=\frac{Z_{\alpha}\left(Z_{\beta}+Z_{\gamma}\right)}{\sqrt{2 \mu_{\alpha(\beta \gamma)}} y_{\alpha}}\left(1-e^{\left(-\frac{y_{\alpha}}{0.05 R_{\alpha}}\right)^{2}}\right) .
$$

The permutational symmetry with respect to electrons have been used in the calculations by reducing the number of Eqs. (3.8) to $2 n_{M}$ for a given orbital momentum $J$.

The Helium trimer is a weakly-bound molecular system that has only two bound states with $J=0$ with extremely small energy values and spatially extended wave functions. Calculations of these energy levels is a well-known computational challenge. We compare our test calculation results with benchmark calculations of [10]. The interatomic interactions are described by the TTY potential and atomic Helium mass is taken to be $M_{\mathrm{He}}=7296.2994$ a.u. To normalize the values of coordinates $x_{\alpha}$ and $y_{\alpha}$ in the calculations we used $1 / M_{\mathrm{He}}$ a.u. as energy units and $\sqrt{M_{\mathrm{He}}}$ a.u. as length units. For $x_{\alpha}$ spline nodes we used the mapping (4.3) and for $y_{\alpha}$

$$
\left(\left(\left(R_{y_{\alpha}}+1\right)^{\frac{1}{3}}-1\right) u(i)+1\right)^{3}-1
$$

with $u(i)=y_{\alpha}(i) / R_{y_{\alpha}}$ and equidistant points $y_{\alpha}(i)$. The permutational symmetry with respect to all three particles in this case implies one Eq. (3.8). 
Table 2: Convergence of the ground state $(J=0, p=1)$ energy level of Helium trimer with respect to basis sizes. The solution domain is $(1: 1200.0,2450.0)$ a.u. The benchmark calculations of [10] give value $-4.00720 \mathrm{e}-7$.

\begin{tabular}{||ll||}
\hline Basis sizes & $E, 10^{-7}$ a.u. \\
\hline$(1: 58,58,12)$ & -2.9142 \\
$(1: 88,88,15)$ & -4.0895 \\
$(1: 118,118,18)$ & -4.0219 \\
$(1: 148,148,21)$ & -4.0070 \\
$(1: 178,178,24)$ & -4.0081 \\
$(1: 208,208,27)$ & -4.0081 \\
$(1: 223,223,27)$ & -4.0077 \\
\hline
\end{tabular}

Table 1 shows comparison of the presented tensor product preconditioner approach and an alternative approach based on applying the Intel MKL Pardiso solver [53] for inverting the matrix $\left(H-E^{*} S\right)$ of (3.10) for IRAM iterations. We note that although the matrix $\left(H-E^{*} S\right)$ is inevitably stored as a sparse matrix in CSR format in the Pardiso approach, the memory allocated for storing its elements values (the known CSR "aa" array of values) is freed right after the matrix has been factorized by Pardiso (phases 1 and 2 of Intel MKL Pardiso [53]). In other words, we tried to do our best to minimize memory usage in the Pardiso approach. However, as is seen from Table 1, memory and CPU time usage grow very fast as the number of basis functions increases.

Fig. 4 and Table 2 demonstrate convergence of energy level values of atomic Helium and molecular Helium trimer with respect to basis sizes. In the calculations, the largest presented basis sets lead to the matrices of linear size 4246848 for atomic Helium and 1342683 for molecular Helium. In both cases, the agreement with benchmark values is good and our universal approach provides a clear convergence to these values. In the simpler case of atomic Helium the relative error of approximately $10^{-8}-10^{-9}$ is reached with basis size that is far from maximal possible one available for our calculations.

Table 3 shows performance of the present paper algorithm for the high $J=10$ case. In this case a set of twenty coupled 3D PDEs (3.8) has been solved. Table 3 shows that the relative error of approximately $10^{-5}$ is reached with relatively small computer resources.

\section{Conclusions}

In this work we have presented the computational approach for solving the quantum three-body problem. It is based on solving the Faddeev-Merkuriev equations in total orbital momentum representation. These are the finite set of coupled three-dimensional PDEs, that are solved by the method of spline collocations. The idea of fast inverting the matrix of the operator at the left-hand side of the FM equations written in tensor product form is used. In our approach it is done in the form of approximate preconditioning. The tensor product form is also used in the matrix storage scheme, which leads to a 


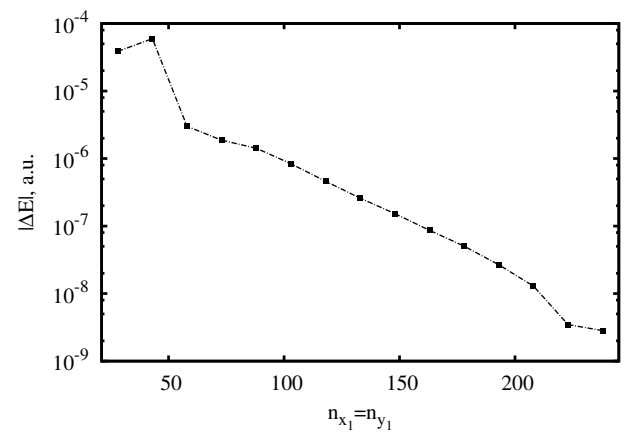

(a)

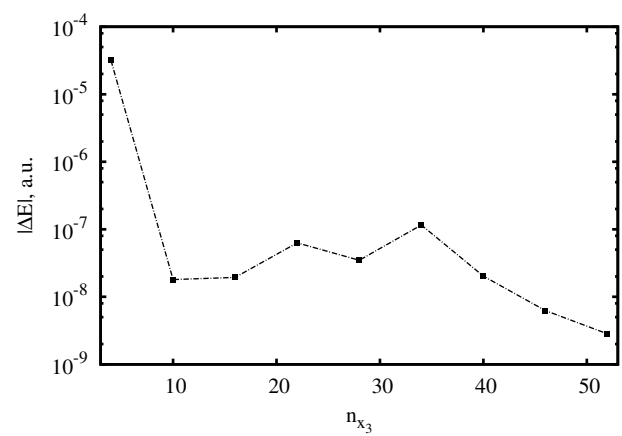

(c)

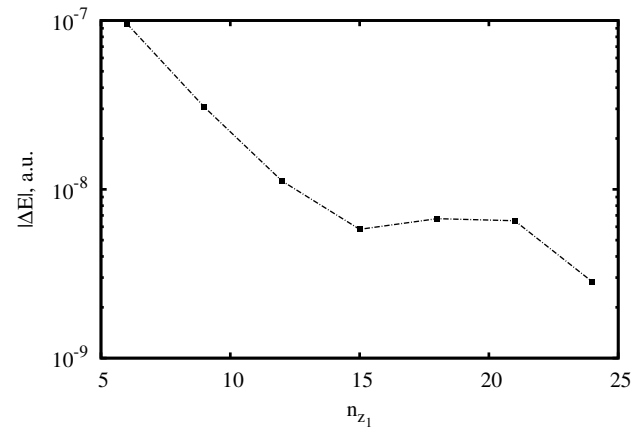

(b)

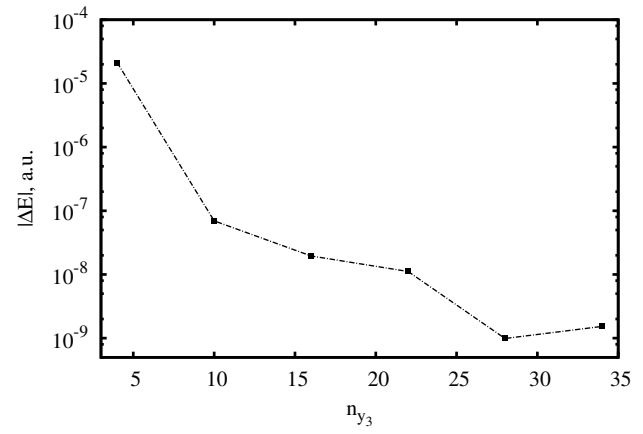

(d)

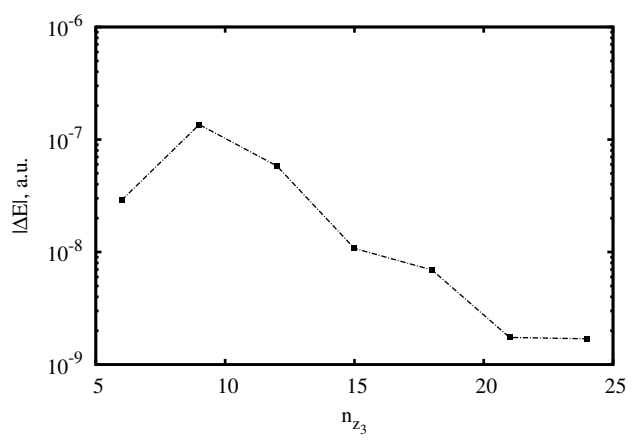

(e)

Figure 4: Convergence of $3^{1} D(J=2, \tau=1, p=1)$ energy level of atomic Helium with respect to basis sizes in different coordinates. In each figure the chosen parameter of the final basis $(1: 238,238,24 ; 3: 52,40,27)$ is being changed. The solution domains are $(1: 45.0,45.0 ; 3: 11.0,6.0)$ a.u. The difference $\Delta E$ is that of the calculated value and the benchmark value -2.05562073285224648939 of [54].

significant economy in both computer resources and time under some assumptions on bases and collocation points. In the calculations, we have shown the universality and efficiency of our approach in solving bound state problems of different nature. In the 
Table 3: Performance of the present paper algorithm for the high $J$ case. The ground and first excited state energies of the Helium atom with $J=10, \tau=1, p=1$ are calculated in each run. The solution domains are ( 1 : $280.0,280.0 ; 3$ : 2.0, 2.0) a.u.

\begin{tabular}{|c|c|c|c|c|c|}
\hline Basis sizes & $\begin{array}{l}\text { Matrix } \\
\text { linear } \\
\text { size }\end{array}$ & $\begin{array}{l}\text { Total } \\
\text { memory/ } \\
H-E^{*} S / \\
\text { Preconditioner } \\
\text { size, GB }\end{array}$ & $\begin{array}{l}\text { Calculate } \\
\text { matrices/ } \\
\text { IRAM } \\
\text { wall } \\
\text { time, } \\
\text { minutes }\end{array}$ & $\begin{array}{l}\text { Total } \\
\text { CPU } \\
\text { time, } \\
\text { minutes }\end{array}$ & $E$, a.u. \\
\hline $\begin{array}{l}(1: 58,58,6 ; \\
3: 4,4,6)\end{array}$ & 223080 & $0.62 / 0.17 / 0.11$ & $0.1 / 6.3$ & 38 & $\begin{array}{l}-2.00398 \\
-2.00332\end{array}$ \\
\hline $\begin{array}{l}(1: 88,88,9 \\
3: 7,7,9)\end{array}$ & 771507 & $2.2 / 0.59 / 0.43$ & $0.7 / 33$ & 200 & $\begin{array}{l}-2.00412 \\
-2.00346\end{array}$ \\
\hline $\begin{array}{l}(1: 118,118,9 \\
3: 7,7,9)\end{array}$ & 1383327 & $4.1 / 1.06 / 1.01$ & $1.9 / 60$ & 350 & $\begin{array}{l}-2.00413 \\
-2.00347\end{array}$ \\
\hline $\begin{array}{l}(1: 118,118,12 \\
3: 7,7,12)\end{array}$ & 1844436 & $7.3 / 1.41 / 1.18$ & $2.4 / 280$ & 1500 & $\begin{array}{l}-2.00413 \\
-2.00347\end{array}$ \\
\hline
\end{tabular}

future, we plan to use it for solving multichannel scattering problems in various systems of three neutral and charged particles.

\section{Acknowledgments}

VAG' s work was supported by Russian Science Foundation grant No. 19-72-00076. VAR, EAY and SLY' s work was supported by Russian Foundation for Basic Research grant No. 18-02-00492. Research was carried out using computational resources provided by Resource Center "Computer Center of SPbU" (http://cc.spbu.ru).

\section{Appendix A}

In this Appendix we clarify the assumptions under which the approximate equalities (3.31) hold. Let us first introduce the eigenfunctions of the operator $\boldsymbol{d}_{z_{\alpha}}^{M^{\prime}}$ defined in (3.24)

$$
g_{\ell}^{M^{\prime}}\left(z_{\alpha}\right)=\sqrt{\frac{2 \ell+1}{2} \frac{\left(\ell-M^{\prime}\right) !}{\left(\ell+M^{\prime}\right) !}} \frac{P_{\ell}^{M^{\prime}}\left(z_{\alpha}\right)}{\left(1-z_{\alpha}^{2}\right)^{M^{\prime} / 2}},
$$

where $P_{\ell}^{M^{\prime}}$ are the associated Legendre polynomials [40]. From their properties it follows that $g_{\ell}^{M^{\prime}} \equiv 0$ when $\ell<M^{\prime}$, otherwise $g_{\ell}^{M^{\prime}}$ is a polynomial of degree $\ell-M^{\prime}$. They satisfy

$$
\begin{aligned}
& \boldsymbol{d}_{z_{\alpha}}^{M^{\prime}} g_{\ell}^{M^{\prime}}\left(z_{\alpha}\right)=-\ell(\ell+1) g_{\ell}^{M^{\prime}}\left(z_{\alpha}\right), \\
& \boldsymbol{d}_{z_{\alpha}}^{M^{\prime} \pm} g_{\ell}^{M^{\prime} \pm 1}\left(z_{\alpha}\right)=\mp \lambda^{\ell, \pm M^{\prime}} g_{\ell}^{M^{\prime}}\left(z_{\alpha}\right),
\end{aligned}
$$


with the operators $\boldsymbol{d}_{z_{\alpha}}^{M^{\prime} \pm}$ from (3.24).

Now we are able to formulate the following statements:

1. If for a given $M^{\prime}=M_{0}, \cdots, J$ the basis of functions $S_{\alpha}^{k}(z)$ is chosen so that it approximates the first $n_{z_{\alpha}}$ eigenfunctions $g_{\ell}^{M^{\prime}}\left(\ell=M^{\prime}, \cdots, M^{\prime}+n_{z_{\alpha}}-1\right)$ and their first two derivatives on the grid of collocation points $z_{\alpha}^{\zeta}$ chosen close to roots of $g_{M^{\prime}+n_{z \alpha}}^{M^{\prime}}$, then the approximate equalities (3.31) hold.

2. If (nonlocal) basis functions $S_{\alpha}^{k}$ are polynomials of degree $k$ and roots of $g_{M^{\prime}+n_{z \alpha}}^{M^{\prime}}$ are chosen as collocation points to represent Eq. (3.8) with given $M^{\prime}$, then approximate equalities (3.31) are exact.

To justify the statements, we note that since by definition (3.25) the equality $D_{z_{\alpha}}^{M^{\prime}} W_{z_{\alpha}}^{M^{\prime}}=$ $S_{z_{\alpha}} W_{z_{\alpha}}^{M^{\prime}} \widetilde{\Lambda}_{z_{\alpha}}^{M^{\prime}}$ holds, $\widetilde{\Lambda}_{z_{\alpha}}^{M^{\prime}}$ is the matrix of approximate eigenvalues and columns of $W_{z_{\alpha}}^{M^{\prime}}$ are the expansion coefficients in terms of basis functions $S_{\alpha}^{k}$ of approximate eigenvectors of the differential eigenvalue problem (A.2), obtained via collocation procedure. Then by assumption 1 the first approximate equality of (3.31) follows. Now the approximate equality

$$
D_{z_{\alpha}}^{M^{\prime}-} W_{z_{\alpha}}^{M^{\prime}-1} \approx S_{z_{\alpha}} W_{z_{\alpha}}^{M^{\prime} \rightarrow} \Lambda_{z_{\alpha}}^{M^{\prime}-\downarrow}
$$

is the consequence of exact equality (A.3) with lower sign. Here the matrix

$$
\Lambda_{z_{\alpha}}^{M^{\prime}-\downarrow}=\operatorname{diag}\left\{\lambda^{M^{\prime}-1,-M^{\prime}}, \lambda^{M^{\prime},-M^{\prime}}, \cdots, \lambda^{M^{\prime}+n_{z_{\alpha}}-2,-M^{\prime}}\right\}
$$

and the matrix $W_{z_{\alpha}}^{M^{\prime}} \rightarrow$ is obtained from $W_{z_{\alpha}}^{M^{\prime}}$ by shifting its columns by one position to the right with replacing first column with zeros and throwing off the last column. Similarly, the approximate equation

$$
D_{z_{\alpha}}^{M^{\prime}+} W_{z_{\alpha}}^{M^{\prime}+1} \approx\left(S_{z_{\alpha}} W_{z_{\alpha}}^{M^{\prime} \leftarrow} \mid \widetilde{g}_{M^{\prime}+n_{z_{\alpha}}}^{M^{\prime}}\right) \Lambda_{z_{\alpha}}^{M^{\prime}+\uparrow}
$$

is the consequence of (A.3) with upper sign. The matrix

$$
\Lambda_{z_{\alpha}}^{M^{\prime}+\uparrow}=\operatorname{diag}\left\{-\lambda^{M^{\prime}+1, M^{\prime}},-\lambda^{M^{\prime}+2, M^{\prime}}, \cdots,-\lambda^{M^{\prime}+n_{z_{\alpha}}, M^{\prime}}\right\}
$$

and the matrix $W_{z_{\alpha}}^{M^{\prime} \leftarrow}$ is obtained from $W_{z_{\alpha}}^{M^{\prime}}$ by shifting its columns by one position to the left with throwing off the first one. The column $\widetilde{g}_{M^{\prime}+n_{z_{\alpha}}}^{M^{\prime}}$ is filled with approximate values of the function $g_{M^{\prime}+n_{z_{\alpha}}}^{M^{\prime}}$ at collocation points. By assumption the approximate equality $\widetilde{g}_{M^{\prime}+n_{z \alpha}}^{M^{\prime}} \approx 0$ is true. Then using (A.4), (A.6) and the definition (3.25) of matrices $\bar{W}_{z_{\alpha}}^{M^{\prime}}, W_{z_{\alpha}}^{M^{\prime}}$ we obtain the remaining approximate equalities

$$
\begin{aligned}
& \bar{W}_{z_{\alpha}}^{M^{\prime}}\left(D_{z_{\alpha}}^{M^{\prime}-} W_{z_{\alpha}}^{M^{\prime}-1}\right) \approx\left(\bar{W}_{z_{\alpha}}^{M^{\prime}} S_{z_{\alpha}} W_{z_{\alpha}}^{M^{\prime} \rightarrow}\right) \Lambda_{z_{\alpha}}^{M^{\prime}-\downarrow}=\Lambda_{z_{\alpha}}^{M^{\prime}-}, \\
& \bar{W}_{z_{\alpha}}^{M^{\prime}}\left(D_{z_{\alpha}}^{M^{\prime}+} W_{z_{\alpha}}^{M^{\prime}+1}\right) \approx\left(\bar{W}_{z_{\alpha}}^{M^{\prime}} S_{z_{\alpha}} W_{z_{\alpha}}^{M^{\prime} \leftarrow} \mid 0\right) \Lambda_{z_{\alpha}}^{M^{\prime}+\uparrow}=\Lambda_{z_{\alpha}}^{M^{\prime}+} .
\end{aligned}
$$


If a polynomial basis is used and collocation points are roots of polynomials $g_{M^{\prime}+n_{z_{\alpha}}}^{M^{\prime}}$, then $W_{z_{\alpha}}^{M^{\prime}}$ is an exact representation of functions $g_{\ell}^{M^{\prime}}$ in this basis and one has $\widetilde{g}_{M^{\prime}+n_{z_{\alpha}}}^{M^{\prime}}=0$. Thus all approximate equalities above are exact.

\section{References}

[1] E. A. Hylleraas. Neue berechnung der energie des heliums im grundzustande, sowie des tiefsten terms von ortho-helium. Z. Physik, 54:347-366, 1929.

[2] L. H. Thomas. The interaction between a neutron and a proton and the structure of $\mathrm{H}^{3}$. Phys. Rev., 47:903-909, 1935.

[3] V. Efimov. Energy levels arising from resonant two-body forces in a three-body system. Phys. Lett. B, 33(8):563-564, 1970.

[4] A. C. Phillips. Consistency of the low-energy three-nucleon observables and the separable interaction model. Nucl. Phys. A, 107(1):209-216, 1968.

[5] V. Roudnev and M. Cavagnero. Approaching universality in weakly bound three-body systems. Phys. Rev. Lett., 108(11):110402, 2012.

[6] R.A. Aziz and M.J. Slaman. An examination of ab initio results for the helium potential energy curve. J. Chem. Phys., 94(12):8047-8053, 1991.

[7] S. M. Cybulski and R. R. Toczyłowski. Ground state potential energy curves for $\mathrm{He}_{2}, \mathrm{Ne}_{2}$, $\mathrm{Ar}_{2}, \mathrm{HeNe}, \mathrm{HeAr}$, and NeAr: A coupled-cluster study. J. Chem. Phys., 111(23):10520-10528, 1999.

[8] M. Jeziorska, W. Cencek, K. Patkowski, B. Jeziorski, and Szalewicz K. Pair potential for helium from symmetry-adapted perturbation theory calculations and from supermolecular data. J. Chem. Phys., 127(12):124303, 2007.

[9] H. Witała, A. Nogga, H. Kamada, W. Glöckle, J. Golak, and R. Skibiński. Modern nuclear force predictions for the neutron-deuteron scattering lengths. Phys. Rev. C, 68:034002, 2003.

[10] V. Roudnev and M. Cavagnero. Benchmark helium dimer and trimer calculations with a public few-body code. J. Phys. B: At. Mol. Opt. Phys., 45(2):025101, 2012.

[11] M. Decker, W. Sandhas, and V. B. Belyaev. Muonic three-body Coulomb systems in the hyperspherical approach. Phys. Rev. A, 53:726-736, 1996.

[12] A. K. Bhatia, A. Temkin, and H. Eiserike. Rigorous precision $p$-wave positron-hydrogen scattering calculation. Phys. Rev. A, 9:219-222, 1974.

[13] J. W. Humberston, P. Van Reeth, M. S. T. Watts, and W. E. Meyerhof. Positron - hydrogen scattering in the vicinity of the positronium formation threshold. J. Phys. B: At. Mol. Opt. Phys., 30(10):2477-2493, 1997.

[14] J. Mitroy and K. Ratnavelu. The positron-hydrogen system at low energies. J. Phys. B: At. Mol. Opt. Phys., 28(2):287-306, 1995.

[15] A. Igarashi and N. Toshima. Hyperspherical coupled-channel study of positronium formation. Phys. Rev. A, 50:232-239, 1994.

[16] S. J. Ward and J. Shertzer. Hyperspherical hidden crossing method applied to Ps(1s)formation in low energy e ${ }^{+}-\mathrm{H}, \mathrm{e}^{+}-\mathrm{Li}$ and $\mathrm{e}^{+}-\mathrm{Na}$ collisions. New J. Phys., 14(2):025003, 2012.

[17] A. A. Kvitsinsky, A. Wu, and C.-Y. Hu. Scattering of electrons and positrons on hydrogen using the Faddeev equations. J. Phys. B: At. Mol. Opt. Phys., 28(2):275-285, 1995.

[18] C.-Y. Hu. The modified Faddeev equation and multichannel positron-hydrogen scattering calculation. J. Phys. B: At. Mol. Opt. Phys., 32(12):3077-3090, 1999. 
[19] Z. Papp, C.-Y. Hu, Z. T. Hlousek, B. Konya, and S. L. Yakovlev. Three-potential formalism for the three-body scattering problem with attractive Coulomb interactions. Phys. Rev. A, 63:062721, 2001.

[20] S. L. Yakovlev, C.-Y. Hu, and D. Caballero. Multichannel formalism for positron-hydrogen scattering and annihilation. J. Phys. B: At. Mol. Opt. Phys., 40(10):1675-1693, 2007.

[21] R. Lazauskas. Application of the complex scaling method in solving three-body Coulomb scattering problem. J. Phys. B: At. Mol. Opt. Phys., 50(5):055201, 2017.

[22] M. V. Volkov, N. Elander, E. Yarevsky, and S. L. Yakovlev. Solving the Coulomb scattering problem using the complex-scaling method. Eur. Phys. Lett., 85(3):30001, 2009.

[23] M. V. Volkov, E. A. Yarevsky, and S. L. Yakovlev. Potential splitting approach to the threebody Coulomb scattering problem. Eur. Phys. Lett., 110(3):30006, 2015.

[24] E. Yarevsky, S. L. Yakovlev, Å. Larson, and N. Elander. Potential-splitting approach applied to the Temkin-Poet model for electron scattering off the hydrogen atom and the helium ion. J. Phys. B: At. Mol. Opt. Phys., 48(11):115002, 2015.

[25] E. Yarevsky, S. L. Yakovlev, and N. Elander. Potential splitting approach to e-H and e-He ${ }^{+}$ scattering. J. Phys. B: At. Mol. Opt. Phys., 50(5):055001, 2017.

[26] L. D. Faddeev. Mathematical aspects of the Three-body Problem in the Quantum Scattering Theory. Daniel Davey, New York, 1965.

[27] S. L. Yakovlev. Faddeev differential equations as a spectral problem for a nonsymmetric operator. Theor. Math. Phys., 107:835-847, 1996.

[28] S. L. Yakovlev. Quantum N-body problem: matrix structures and equations. Theor. Math. Phys., 181:1317-1338, 2014.

[29] S. P. Merkuriev. On the three-body Coulomb scattering problem. Ann. Phys., 130(2):395-426, 1980.

[30] L. D. Faddeev and S. P. Merkuriev. Quantum Scattering Theory for Several Particle Systems. Kluwer, Dordrech, 1993.

[31] S. L. Yakovlev and Z. Papp. The three-body Coulomb scattering problem in a discrete Hilbert-space basis representation. Theor. Math. Phys., 163:666-676, 2010.

[32] A. A. Kvitsinsky, J. Carbonell, and C. Gignoux. Faddeev calculation of $e^{-}$-Ps scattering lengths. Phys. Rev. A, 46:1310-1315, 1992.

[33] V. A. Gradusov, V. A. Roudnev, E. A. Yarevsky, and S. L. Yakovlev. High resolution calculations of low energy scattering in $\mathrm{e}^{-} \mathrm{e}^{+} \overline{\mathrm{p}}$ and $\mathrm{e}^{+} \mathrm{e}^{-} \mathrm{He}^{++}$systems via Faddeev-Merkuriev equations. J. Phys. B: At. Mol. Opt. Phys., 52(5):055202, 2019.

[34] V. A. Gradusov, V. A. Roudnev, and S. L. Yakovlev. Merkuriev cut-off in $\mathrm{e}^{+}-\mathrm{H}$ multichannel scattering calculations. Atoms, 4(1):9, 2016.

[35] N. W. Schellingerhout, L. P. Kok, and G. D. Bosveld. Configuration-space Faddeev calculations: Supercomputer accuracy on a personal computer. Phys. Rev. A, 40:5568-5576, 1989.

[36] Rimantas Lazauskas. Etude de la diffusion de particules lourdes sur des systèmes atomiques et nucléaires. PhD thesis, 2003. https://hal . archives-ouvertes .fr/tel-00004178.

[37] V. V. Kostrykin, A. A. Kvitsinsky, and S. P. Merkuriev. Faddeev approach to the three-body problem in total-angular-momentum representation. Few Body Syst., 6:97-113, 1989.

[38] A. Messiah. Quantum Mechanics. North-Holland, Amsterdam, 1961.

[39] Wolfram Research, Inc. Mathematica, Version 9.0. https://www.wolfram.com/ mathematica, 2012.

[40] NIST Digital Library of Mathematical Functions. http://dlmf .nist.gov/,2019.

[41] L. C. Biedenharn and J. D. Louck. Angular Momentum in Quantum Physics. Addison-Wesley, Reading, MA, 1981. 
[42] D. A. Varshalovich, A. N. Moskalev, and V. K. Khersonsky. Quantum Theory of Angular Momentum. World Scientific, Singapore, 1989.

[43] A. Scrinzi. Helium in a cylindrically symmetric field. J. Phys. B: At. Mol. Opt. Phys., 29(24):60556068, 1996.

[44] V. Roudnev and S. Yakovlev. Improved tensor-trick algorithm: application to helium trimer. Comput. Phys. Comm., 126(1):162-164, 2000.

[45] B. Bialecki, G. Fairweather, and A. Karageorghis. Matrix decomposition algorithms for elliptic boundary value problems: a survey. Numer. Algor., 56:253-295, 2011.

[46] Y. Saad. Iterative Methods for Sparse Linear Systems. SIAM, Philadelphia, 2003.

[47] B. Bialecki and G. Fairweather. Orthogonal spline collocation methods for partial differential equations. J. Comput. Appl. Math., 128(1):55-82, 2001. Numerical Analysis 2000. Vol. VII: Partial Differential Equations.

[48] Alexey Kornev. Research project, 2019.

[49] C. de Boor and B. Swartz. Collocation at gaussian points. SIAM J. Numer. Anal., 10(4):582606, 1973.

[50] D. C. Sorensen. Implicit application of polynomial filters in a $k$-step Arnoldi method. SIAM J. Matrix Anal. Appl., 13:357-385, 1992.

[51] Y. Saad. Numerical Methods for Large Eigenvalue Problems. SIAM, Philadelphia, 2011.

[52] R. B. Lehoucq, D. C. Sorensen, and C. Yang. ARPACK USERS GUIDE: Solution of Large Scale Eigenvalue Problems by Implicitly Restarted Arnoldi Methods. SIAM, Philadelphia, 1998.

[53] Intel Math Kernel Library Developer Reference. https://software.intel.com/en-us/ mkl-developer-reference-c, 2019.

[54] D. T. Aznabayev, A. K. Bekbaev, I. S. Ishmukhamedov, and V. I. Korobov. Energy levels of a helium atom. Phys. Part. Nucl. Lett., 12:689-694, 2015.

[55] A. A. Kvitsinsky and C.-Y. Hu. Zero-energy scattering in symmetric Coulomb systems via Faddeev approach. Phys. Rev. A, 47:994-999, 1993. 